\title{
Linking pre- and proto-stellar objects in the intermediate-/high-mass star forming region IRAS 05345+3157
}

\author{
F. Fontani ${ }^{1,2}$, Q. Zhang ${ }^{3}$, P. Caselli ${ }^{4}$, and T. L. Bourke ${ }^{3}$ \\ 1 ISDC, Ch. d'Ecogia 16, 1290 Versoix, Switzerland \\ e-mail: Francesco.Fontani@unige.ch \\ 2 Observatoire de Genève, University of Geneva, Ch. de Maillettes 51, 1290 Sauverny, Switzerland \\ 3 Harvard-Smithsonian Center for Astrophysics, 60 Garden Street, Cambridge, MA 02138, USA \\ 4 School of Physics and Astronomy, University of Leeds, Leeds, LS2 9JT, UK
}

Received 2 January 2009 / Accepted 25 February 2009

\section{ABSTRACT}

Context. To better understand the initial conditions of the high-mass star formation process, it is crucial to study at high angular resolution the morphology, the kinematics, and the interactions of the coldest condensations associated with intermediate-/high-mass star forming regions.

Aims. This paper studies the cold condensations in the intermediate-/high-mass proto-cluster IRAS $05345+3157$, focusing on the interaction with the other objects in the cluster.

Methods. We performed millimeter high-angular resolution observations, both in the continuum and several molecular lines, with the PdBI and the SMA. In a recent paper, we published part of these data. The main finding of that work was the detection of two cold and dense gaseous condensations, called $\mathrm{N}$ and $\mathrm{S}$ (masses $\sim 2$ and $\sim 9 M_{\odot}$ ), characterised by high values of deuterium fractionation $(\sim 0.1$ in both cores) obtained from the column density ratio $N\left(\mathrm{~N}_{2} \mathrm{D}^{+}\right) / N\left(\mathrm{~N}_{2} \mathrm{H}^{+}\right)$. In this paper, we present a full report of the observations, and a complete analysis of the data obtained.

Results. The millimeter maps reveal the presence of 3 cores inside the interferometer primary beam, called C1-a, C1-b and C2. None of them are associated with cores $\mathrm{N}$ and S. C1-b is very likely associated with a newly formed early-B ZAMS star embedded inside a hot core, while $\mathrm{C} 1-\mathrm{a}$ is more likely associated with a class 0 intermediate-mass protostar. The nature of $\mathrm{C} 2$ is unclear. Both $\mathrm{C} 1-\mathrm{a}$ and $\mathrm{C} 1-\mathrm{b}$ are good candidates as driving sources of a powerful ${ }^{12} \mathrm{CO}$ outflow, which strongly interacts with $\mathrm{N}$, as demonstrated by the velocity gradient of the gas along this condensation. The $\mathrm{N}_{2} \mathrm{H}^{+}$linewidths are between $\sim 1$ and $2 \mathrm{~km} \mathrm{~s}^{-1}$ in the region where the continuum cores are located, and smaller $\left(\sim 0.5-1.5 \mathrm{~km} \mathrm{~s}^{-1}\right)$ towards $\mathrm{N}$ and $\mathrm{S}$, indicating that the gas in the deuterated condensations is more quiescent than that associated with the continuum sources. This is consistent with the fact that they are still in the pre-stellar phase and hence the star formation process has not yet taken place there.

Conclusions. The study of the gas kinematics across the source indicates a tight interaction between deuterated condensations and the sources embedded in millimeter cores. For the nature of $\mathrm{N}$ and $\mathrm{S}$, we propose two scenarios: they can be low-mass pre-stellar condensations or "seeds" of future high-mass star(s). However, from these data it is not possible to establish how the turbulence triggered by the neghbouring cluster of protostars can influence the evolution of the condensations.

Key words. stars: formation - ISM: molecules

\section{Introduction}

The initial conditions of star formation are still a matter of debate. Studies have begun to unveil the chemical and physical properties of starless low-mass cores on the verge of forming low-mass stars (Kuiper et al. 1996; Caselli et al. 2002a,b; Tafalla et al. 2002, 2006), demonstrating that in the dense and cold

\footnotetext{
* Based on observations carried out with the IRAM Plateau de Bure Interferometer. IRAM is supported by INSU/CNRS (France), MPG (Germany) and IGN (Spain).

$\star \star$ The Submillimeter Array is a joint project between the Smithsonian Astrophysical Observatory and the Academia Sinica Institute of Astronomy and Astrophysics, and is funded by the Smithsonian Institution and the Academia Sinica.

$\star \star \star$ The fits-files of the $93 \mathrm{GHz}$ continuum image and of the $\mathrm{N}_{2} \mathrm{H}^{+}(1-$ 0 ) data-cubes are only available in electronic form at the CDS via anonymous ftp to cdsarc.u-strasbg.fr (130.79.128.5) or via http://cdsweb.u-strasbg.fr/cgi-bin/qcat?J/A+A/499/233
}

nuclei of these cores, $\mathrm{C}$-bearing molecular species such as $\mathrm{CO}$ and CS are strongly depleted (e.g. Caselli et al. 2002b; Tafalla et al. 2002), while N-bearing molecular ions such as $\mathrm{N}_{2} \mathrm{D}^{+}$and $\mathrm{N}_{2} \mathrm{H}^{+}$(Caselli et al. 2002a,b; Crapsi et al. 2005) maintain high abundances in the gas phase, and their column density ratios reach values of $\sim 0.1$ or more, much higher than the cosmic $[\mathrm{D} / \mathrm{H}]$ elemental abundance $\left(\sim 10^{-5}\right.$, Oliveira et al. 2003).

The characterisation of the earliest stages of the formation process of high-mass stars is more difficult than for low-mass objects, given their shorter evolutionary timescales, larger distances, and strong interaction with their environments. Nevertheless, significant progress has been made by various authors who have performed extensive studies aimed at the identification of massive protostellar candidates, i.e. very young $\left(<10^{5} \mathrm{yr}\right)$, massive $\left(M>8 M_{\odot}\right)$ stellar objects which have not yet ionised the surrounding medium (Molinari et al. 1996; Sridharan et al. 2002; Fontani et al. 2005). On the other hand, the pre-protostellar stage, namely the phase in which a cold 
starless core evolves towards the onset of the formation of a high-mass protostar, has not yet been investigated in great detail, even though in the last few years an increasing effort has been devoted to this study (see e.g. Wang et al. 2006; Zhang et al. 2009; Beuther \& Sridharan 2007).

Many ultracompact (UC) HII regions are located in clusters (see e.g. Thompson et al. 2006), and it is common to find massive young stellar objects in different evolutionary stages in the same star forming region (Kurtz et al. 2000). Therefore, one expects to find even earlier phases of massive star formation in the vicinity of UC HII regions and/or other "signposts" of massive star formation activity. With this in mind, several authors have searched for cold and dense spots in the neighbourhood of luminous IRAS point sources (Fontani et al. 2006), UC HII regions (Pillai et al. 2007) and methanol masers (Hill et al. 2006). In particular, Fontani et al. (2006) searched for $\mathrm{N}_{2} \mathrm{D}^{+}$emission in the gas associated with 10 luminous IRAS sources with the IRAM-30 m telescope, and detected deuterated gas in 7 out of the 10 sources observed.

The subject of the present work is one of the sources studied by Fontani et al. (2006), which stands out for its very interesting characteristics: it is a high-mass $\left(\sim 180 M_{\odot}\right.$, Fontani et al. 2006) dusty clump, undetected in the MSX $8 \mu \mathrm{m}$ band, located near the luminous IRAS point source $05345+3157\left(\sim 60^{\prime \prime}\right.$ to the N-E). The region is located at a distance of $1.8 \mathrm{kpc}$ (Zhang et al. 2005), and its surface density $\left(\sim 1.3 \mathrm{~g} \mathrm{~cm}^{-2}\right)$ and mass-to-luminosity ratio $\left(\sim 8 L_{\odot} / M_{\odot}\right)$ indicate that it is potentially a site of massive star formation (see Fig. 1 of Chakrabarti \& McKee 2005). Hereafter, we will call our target I05345. Previous interferometric studies have reavealed a clumpy structure of the molecular gas in I05345 (Molinari et al. 2002). From IRAM-30 m data, Fontani et al. (2006) have measured an average CO depletion factor (ratio between expected and observed $\mathrm{CO}$ abundance) of $\sim 3$ and an average deuterium fractionation (the column density ratio between a deuterated species and the corresponding one containing hydrogen) of $\sim 0.01$, three orders of magnitude higher than the cosmic $[\mathrm{D} / \mathrm{H}]$ abundance. These findings indicate for the first time the possible presence of a high-mass pre-stellar core (with $T \sim 10 \mathrm{~K}$ and $n_{\mathrm{H}_{2}} \sim 10^{6} \mathrm{~cm}^{-3}$ ), analogous to those detected in several low-mass star forming regions. However, the angular resolution was insufficient to determine whether we are dealing with a single high-mass core or instead with a sample of lowmass ones.

Therefore, we recently mapped I05345 at high-angular resolution in the $\mathrm{N}_{2} \mathrm{H}^{+}(1-0)$ line with the IRAM Plateau de Bure Interferometer (PdBI), and in the $\mathrm{N}_{2} \mathrm{H}^{+}$and $\mathrm{N}_{2} \mathrm{D}^{+}(3-2)$ lines with the Submillimeter Array (SMA), in order to derive a detailed map of the deuterium fractionation in the source. Simultaneously, we obtained observations in the continuum at $\sim 96, \sim 225$ and $\sim 284 \mathrm{GHz}$ with the two interferometers, as well as in several lines of other molecules. The preliminary results of these observations, focused mainly on the deuterium fractionation derived from the $\mathrm{N}_{2} \mathrm{H}^{+}$and $\mathrm{N}_{2} \mathrm{D}^{+}$data, have been published by Fontani et al. (2008, hereafter Paper I). For completeness, in Fig. 1 we summarise the main observational results of that work, by showing: (i) the $3 \mathrm{~mm}$ continuum observed with PdBI, which reveals the presence of 4 cores, one of which lies outside the interferometer primary beam; (ii) the distribution of the intensity of the $\mathrm{N}_{2} \mathrm{H}^{+}$(1-0) line, which is extended and has a complex structure; (iii) the distribution of the $\mathrm{N}_{2} \mathrm{D}^{+}$(3-2) line integrated emission, concentrated in two condensations, called $\mathrm{N}$ and $\mathrm{S}$. The integrated emission of the $\mathrm{N}_{2} \mathrm{H}^{+}(3-2)$ line (not shown in Fig. 1) is compact, and is detected towards the strongest continuum peak only. In Paper I we derived

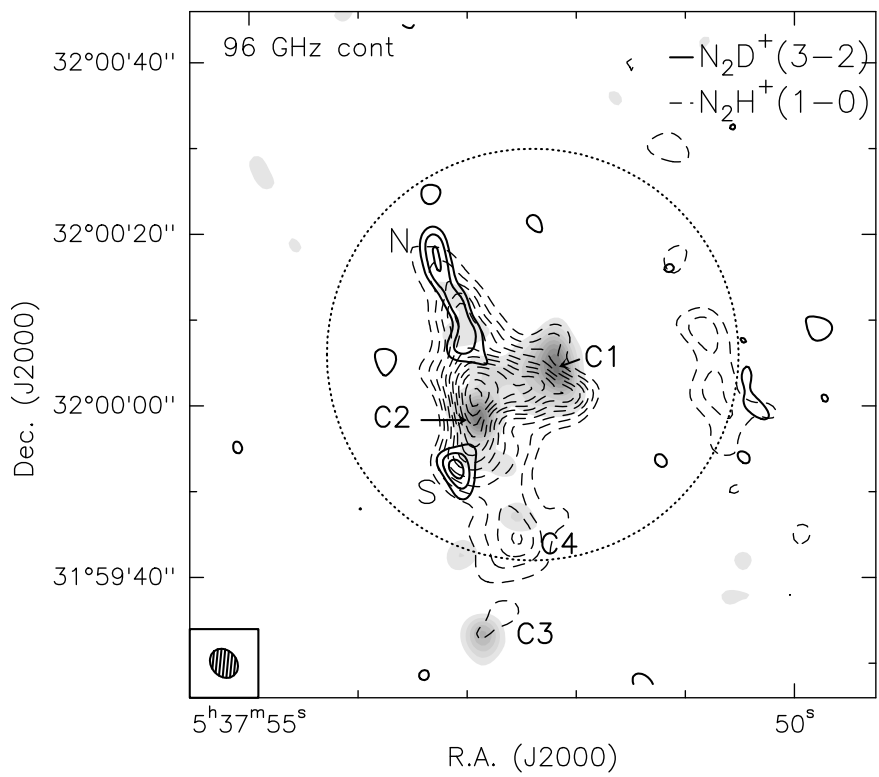

Fig. 1. Summary of the main findings of Paper I: the dashed contours represent the intensity of the $\mathrm{N}_{2} \mathrm{H}^{+}(1-0)$ line integrated between -21.2 and $-14.5 \mathrm{~km} \mathrm{~s}^{-1}$, corresponding to the main group of the hyperfine components of this line, observed with the PdBI (see also Fig. 2 of Paper I). Levels range from the $3 \sigma \mathrm{rms}$, which is $\sim 0.02 \mathrm{Jy} \mathrm{beam}^{-1}$, to $0.26 \mathrm{Jy} \mathrm{beam}^{-1}$, in steps of $3 \sigma$. The grey scale shows the $96 \mathrm{GHz}$ continuum: the levels range from the $3 \sigma \mathrm{rms}\left(4.2 \times 10^{-4} \mathrm{Jy} \mathrm{beam}^{-1}\right)$ to $4 \times 10^{-3} \mathrm{Jy}$ beam $^{-1}$, in steps of $3 \sigma$. The solid contours represent the $\mathrm{N}_{2} \mathrm{D}^{+}(3-2)$ line emission integrated between -18.37 and $-16.7 \mathrm{~km} \mathrm{~s}^{-1}$, obtained with the SMA (see also Fig. 1 of Paper I). The two main condensations are indicated as $\mathrm{N}$ and $\mathrm{S}$. Contour levels start from the $3 \sigma$ rms $\left(\sim 0.09 \mathrm{Jy} \mathrm{beam}^{-1}\right)$, and are in steps of $2 \sigma$. The $96 \mathrm{GHz}$ continuum cores are indicated as $\mathrm{C} 1, \mathrm{C} 2, \mathrm{C} 3$ and $\mathrm{C} 4$. The dotted circle represents the PdBI primary beam at $96 \mathrm{GHz}\left(\sim 48^{\prime \prime}\right)$. The ellipse in the bottom left corner shows the synthesised beam of the $\mathrm{N}_{2} \mathrm{D}^{+}$image, comparable to that of the PdBI at $96 \mathrm{GHz}$.

the masses of $\mathrm{N}$ and $\mathrm{S}$, which are $\sim 9$ and $\sim 2.5 M_{\odot}$, respectively. Also, from the $\mathrm{N}_{2} \mathrm{D}^{+} / \mathrm{N}_{2} \mathrm{H}^{+}$column density ratio we obtained a deuterium fractionation of $\sim 0.1$ in both condensations, which is the typical value derived in low-mass pre-stellar cores.

In this work we present a full report of the observations presented in Paper I, and a complete analysis of the data obtained. In Sect. 2 we describe the observations and the data reduction, while the results are presented in Sect. 3. In Sect. 4 we derive the main physical parameters, which are then discussed in Sect. 5. The main findings of this work are summarised in Sect. 6.

\section{Observations and data reduction}

\subsection{Submillimeter Array observations}

Observations of $\mathrm{N}_{2} \mathrm{D}^{+}(3-2)$ (at $231321 \mathrm{MHz}$ ) and $\mathrm{N}_{2} \mathrm{H}^{+}$(3-2) (at $279511.7 \mathrm{MHz}$ ) towards $\mathrm{I} 05345$ were carried out with the SMA (Ho et al. 2004) in the compact configuration on 30 January and 21 February 2007, respectively. The correlator was configured to observe the continuum emission and several other molecular lines simultaneously (see Table 1). The phase centre was the nominal position of the sub-mm peak detected with the JCMT (Fontani et al. 2006), namely RA(J2000) = $05^{\mathrm{h}} 37^{\mathrm{m}} 52.4^{\mathrm{s}}$ and $\operatorname{Dec}(\mathrm{J} 2000)=32^{\circ} 00^{\prime} 06^{\prime \prime}$, and the local standard of rest velocity $V_{\mathrm{LSR}}$ is $-18.4 \mathrm{~km} \mathrm{~s}^{-1}$. For gain calibrations, observations of I05345 were alternated with the quasars 3C 111 and J0530+135. Quasar 3C 279 and Callisto were used for passband and flux calibration, respectively. The SMA data 
Table 1. Molecular transitions observed with the SMA and the PdBI.

\begin{tabular}{|c|c|c|c|c|}
\hline Transition & $\begin{array}{c}v \\
(\mathrm{GHz})\end{array}$ & $\begin{array}{c}\text { Spec. Resol. } \\
\left(\mathrm{km} \mathrm{s}^{-1}\right)\end{array}$ & Detection & $\begin{array}{c}3 \sigma^{a} \\
\left(\mathrm{Jy} \mathrm{beam}^{-1}\right)\end{array}$ \\
\hline \multicolumn{5}{|c|}{ SMA } \\
\hline $\mathrm{C}^{18} \mathrm{O}(2-1)$ & 219.560 & 0.55 & $\mathrm{Y}$ & 0.18 \\
\hline HNCO (10-9) & 219.798 & 0.55 & $\mathrm{~N}$ & \\
\hline $\mathrm{H}_{2} \mathrm{C}^{13} \mathrm{O}\left(3_{1,2}-2_{1,1}\right)$ & 219.909 & 0.55 & $\mathrm{~N}$ & \\
\hline $\mathrm{SO}\left(5_{6}-4_{5}\right)$ & 219.949 & 0.55 & $\mathrm{Y}$ & 0.12 \\
\hline $\mathrm{CH}_{3} \mathrm{OH}\left(8_{0,3}-7_{1,3}\right)$ & 220.079 & 0.55 & $\mathrm{Y}$ & 0.15 \\
\hline $\mathrm{HCOOCH}_{3}(17-16)$ & 220.167 & 0.55 & $\mathrm{~N}$ & \\
\hline${ }^{13} \mathrm{CO}(2-1)$ & 220.399 & 0.55 & $\mathrm{Y}$ & 0.16 \\
\hline $\mathrm{CH}_{3} \mathrm{CN}(12-11)$ & $220.539-220.747^{b}$ & 0.55 & $\mathrm{Y}$ & 0.15 \\
\hline $\mathrm{HC}_{5} \mathrm{~N}(83-82)$ & 220.937 & 0.55 & $\mathrm{~N}$ & \\
\hline $\mathrm{CH}_{3} \mathrm{OH}\left(8_{-1,8}-7_{0,7}\right)$ & 229.759 & 0.53 & $\mathrm{Y}$ & 0.18 \\
\hline $\mathrm{CH}_{3} \mathrm{OH}\left(3_{2,4}-4_{1,4}\right)$ & 230.027 & 0.53 & $\mathrm{~N}$ & \\
\hline${ }^{12} \mathrm{CO}(2-1)$ & 230.538 & 1.0 & $\mathrm{Y}$ & 0.15 \\
\hline OCS (19-18) & 231.062 & 0.50 & $\mathrm{Y}$ & 0.21 \\
\hline${ }^{13} \mathrm{CS}(5-4)$ & 231.220 & 0.50 & Y & 0.21 \\
\hline $\mathrm{CH}_{3} \mathrm{OH}\left(10_{2,2}-9_{3,2}\right)$ & 231.286 & 0.50 & $\mathrm{~N}$ & \\
\hline $\mathrm{N}_{2} \mathrm{D}^{+}(3-2)$ & 231.321 & 0.52 & $\mathrm{Y}$ & 0.21 \\
\hline $\mathrm{CH}_{3} \mathrm{OH}\left(9_{1,9}-8_{0,8}\right)$ & 278.305 & 0.44 & $\mathrm{~N}$ & \\
\hline $\mathrm{N}_{2} \mathrm{H}^{+}(3-2)$ & 279.512 & 0.44 & $\mathrm{Y}$ & 1.0 \\
\hline OCS (23-22) & 279.685 & 0.44 & $\mathrm{~N}$ & \\
\hline $\mathrm{DCO}^{+}(4-3)$ & 288.144 & 0.44 & $\mathrm{~N}$ & \\
\hline $\operatorname{DCN}(4-3)$ & 288.664 & 0.44 & $\mathrm{~N}$ & \\
\hline$C^{34} S(6-5)$ & 289.209 & 0.44 & $\mathrm{~N}$ & \\
\hline $\mathrm{CH}_{3} \mathrm{OH}(6-5)$ & $289.307-290.939^{c}$ & 0.44 & $\mathrm{~N}$ & \\
\hline $\operatorname{SiS}(16-15)$ & 290.380 & 0.44 & $\mathrm{~N}$ & \\
\hline \multicolumn{5}{|c|}{$\mathrm{PdB}$} \\
\hline
\end{tabular}

\footnotetext{
${ }^{a}$ For the detected transitions only.

${ }^{b}$ Minimum and maximum frequencies corresponding to the components $K=7$ and $K=0$, respectively.

${ }^{c}$ Minimum and maximum frequencies corresponding to the components $\left(6_{0,6}-5_{0,5}\right)$ and $\left(6_{+2,4}-5_{+2,3}\right)$, respectively.
}

were calibrated with the MIR package (Qi 2005), and imaged with MIRIAD (Sault et al. 1995). Channel maps were created with natural weighting, attaining a resolution of: $3^{\prime \prime} \cdot 7 \times 33^{\prime \prime} 0$ for the $\mathrm{N}_{2} \mathrm{D}^{+}(3-2)$ channel map; 3'. $0 \times 2$ 2.' 8 for the $225 \mathrm{GHz}$ continuum image; 2 .' $7 \times 2$ '. 0 for the $\mathrm{N}_{2} \mathrm{H}^{+}(3-2)$ channel map; $1^{\prime \prime} .9 \times 11^{\prime \prime} .2$ for the $284 \mathrm{GHz}$ continuum image. At the assumed distance of $1.8 \mathrm{kpc}$, these values translate into spatial resolutions of $\sim 0.025$ and $\sim 0.01 \mathrm{pc}$ at $225 \mathrm{GHz}$ and $284 \mathrm{GHz}$, respectively (i.e. 5000 and $2000 \mathrm{AU}$, respectively). The $3 \sigma$ level in the 225 and $284 \mathrm{GHz}$ continuum images is 0.006 and $0.018 \mathrm{Jy}^{\text {beam }}{ }^{-1}$, respectively.

The spectral resolution in velocity for the $\mathrm{N}_{2} \mathrm{D}^{+}$and $\mathrm{N}_{2} \mathrm{H}^{+}(3-2)$ was $\sim 0.53$ and $\sim 0.44 \mathrm{~km} \mathrm{~s}^{-1}$, respectively. All the lines observed are listed in Table 1: in Cols. 1 and 2 we give the transitions observed and the line rest frequency, respectively; Col. 3 gives the spectral resolution; Col. 4 reports on the detection (Y) or non-detection (N) of the transition, and the $3 \sigma$ level in the channel maps of the detected transitions is given in Col. 5.

\subsection{Plateau de Bure Interferometer observations}

We observed the $\mathrm{N}_{2} \mathrm{H}^{+}(1-0)$ line at $93173.7725 \mathrm{MHz}$ towards I05345 with the PdBI on 11 and 20 August 2006, in the D configuration, and on 3 April 2007 in the C configuration. In Table 1, the spectral resolution in velocity is given. We used the same phase reference and $V_{\mathrm{LSR}}$ velocities as for the SMA observations. The nearby point sources $0507+179$ and $0552+398$ were used as phase calibrators, while bandpass and flux scale were calibrated from observations of $3 \mathrm{C} 345$ and MWC 349, respectively. For continuum measurements, we placed two $320 \mathrm{MHz}$ correlator units in the band when making the observations in D configuration, and six $320 \mathrm{MHz}$ correlator units in $\mathrm{C}$ configuration. The $\mathrm{N}_{2} \mathrm{H}^{+}$lines were excluded in averaging these units to produce the final continuum image (at $\sim 96095 \mathrm{MHz}$ ). The synthesised beam size of the $\mathrm{N}_{2} \mathrm{H}^{+}$channel map was 3 '. $2 \times 3$ '.' 4 , while that of the continuum was 3 '. $1 \times 3$ '. 2 , which translates into a spatial resolution of $\sim 0.03 \mathrm{pc}$. The $3 \sigma$ level in the continuum image is $4.2 \times 10^{-4} \mathrm{Jy} \mathrm{beam}^{-1}$. We stress that the observations of the $\mathrm{N}_{2} \mathrm{H}^{+}(1-0)$ and $\mathrm{N}_{2} \mathrm{D}^{+}$(3-2) lines have approximately the same angular resolution. The data were reduced with the GILDAS software, developed at IRAM and the Observatoire de Grenoble.

\subsection{Combining SMA and NRAO data for ${ }^{12} \mathrm{CO}(2-1)$}

The problem of the missing short spacing information on the SMA data of the ${ }^{12} \mathrm{CO}(2-1)$ line can be solved combining the SMA data with the peviously published ${ }^{12} \mathrm{CO}(2-1)$ map from the NRAO-12 $\mathrm{m}$ telescope (Zhang et al. 2005). For observing details on the single-dish observations we refer to the paper by Zhang et al. (2005). The single-dish data were converted to visibilities in the MIRIAD package with the task UVMODEL. The interferometer and single-dish data were then processed together. The synthesised beam of the combined data is $2.87^{\prime \prime} \times 3.60^{\prime \prime}\left(\mathrm{PA} \sim 42^{\circ}\right)$. For more details on the method adopted see e.g. Zhang et al. (2007).

\subsection{Data reduction}

The $\mathrm{N}_{2} \mathrm{H}^{+}(1-0)$, (3-2), and $\mathrm{N}_{2} \mathrm{D}^{+}$(3-2) rotational transitions have hyperfine structures. To take this into account, we fitted the 


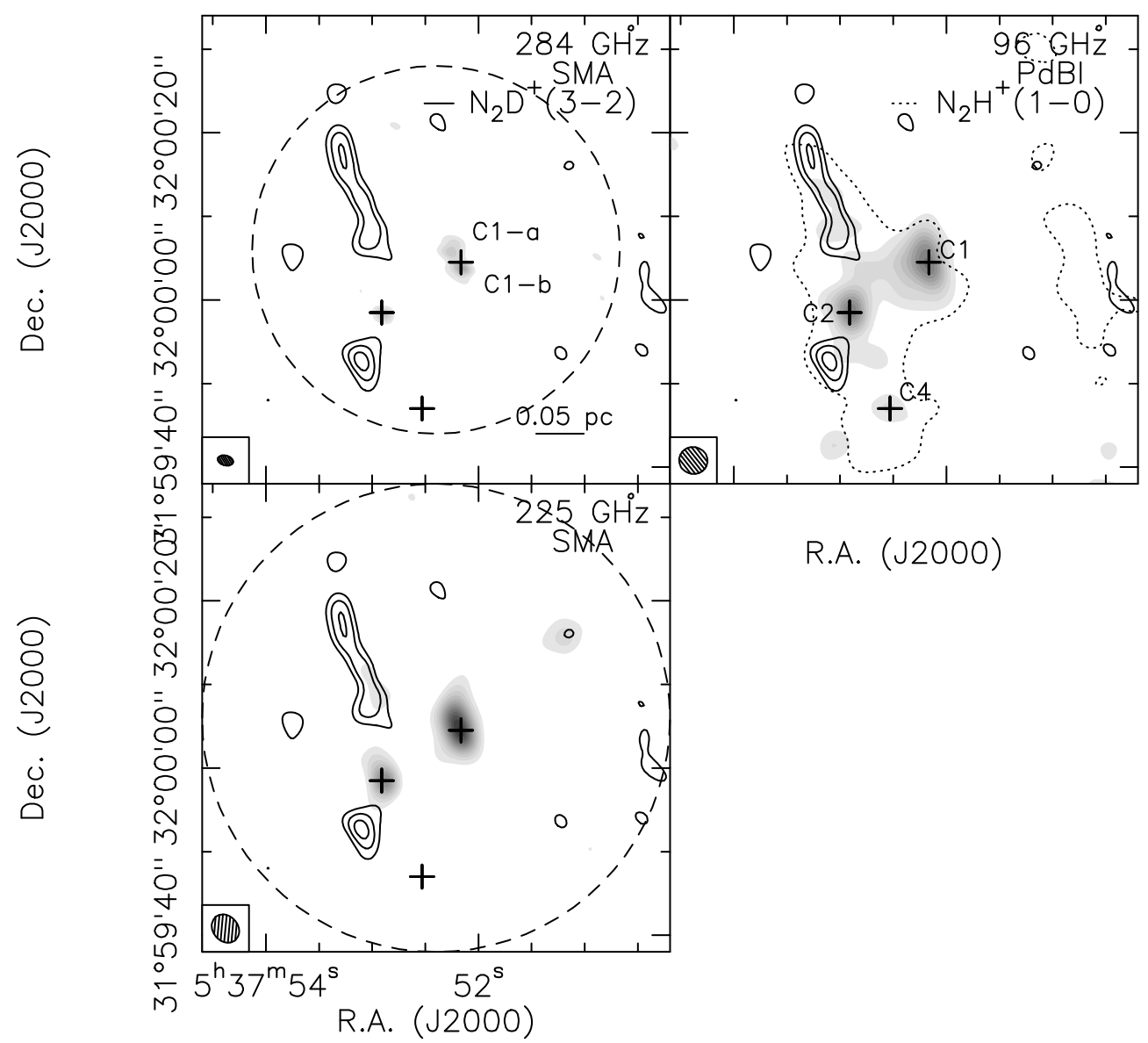

Fig. 2. Top left panel: map of the $284 \mathrm{GHz}$ continuum (grey scale) obtained with the Submillimeter Array. The first level is the $3 \sigma$ rms $=$ $0.018 \mathrm{Jy}_{\text {beam }}{ }^{-1}(\mathrm{step}=3 \sigma \mathrm{rms})$. The crosses indicate the position of the $96 \mathrm{GHz}$ continuum sources shown in Fig. 1 , except C3 which is outside the interferometers primary beam. The thick contours correspond to the integrated emission of the $\mathrm{N}_{2} \mathrm{D}^{+}(3-2)$ line, starting from the $3 \sigma$ level and separated by $2 \sigma$, and thus indicate the position of the pre-stellar cores detected in Paper I (see Fig. 1). The ellipse in the bottom left corner represents the synthesized beam of the continuum image. Bottom left panel: same as top left for the $225 \mathrm{GHz}$ image. The contours start from the $3 \sigma \mathrm{rms}\left(=0.006 \mathrm{Jy} \mathrm{beam}^{-1}\right)$, and are in steps of $3 \sigma$. Right panel: same as left panel for the $96 \mathrm{GHz}$ continuum, observed with the PdBI. The levels are the same as in Fig. 1. The dotted contour corresponds to the $3 \sigma$ contour level of the $\mathrm{N}_{2} \mathrm{H}^{+}(1-0)$ integrated map shown in Fig. 1 .

lines using METHOD HFS of the CLASS program, which is part of the GAG software developed at IRAM and the Observatoire de Grenoble. This method assumes that all the hyperfine components have the same excitation temperature and width, and that their separation is fixed to the laboratory value. The method also provides an estimate of the total optical depth of the lines, based on the intensity ratio of the different hyperfine components. For the rest frequencies of the $\mathrm{N}_{2} \mathrm{H}^{+}$and $\mathrm{N}_{2} \mathrm{D}^{+}$lines, we used the laboratory values given in Crapsi et al. (2005). We have not applied the corrections given in Pagani et al. (2008), since these are well below the spectral resolution of our observations.

\section{Results}

\subsection{Continuum maps}

The 284 and $225 \mathrm{GHz}$ continuum images obtained with the SMA are shown in the top and bottom left panels of Fig. 2, respectively. For a complete comparison among the three continuum maps, in Fig. 2 we also show the continuum emission at $96 \mathrm{GHz}$, already presented in Fig. 1.

At $284 \mathrm{GHz}$, three main compact cores are detected inside the SMA primary beam $\left(44^{\prime \prime}\right)$, two of them close to the map centre and the other one is $\sim 10^{\prime \prime} \mathrm{S}-\mathrm{E}$ of the map centre. We will call the central condensations $\mathrm{C} 1-\mathrm{a}$ and $\mathrm{C} 1-\mathrm{b}$, respectively, because they are located at the position of source $\mathrm{C} 1$ in the $96 \mathrm{GHz}$ image, while the eastern one exactly matches the emission peak of the core $\mathrm{C} 2$. Among the other $96 \mathrm{GHz}$ continuum sources shown in Fig. 1, we clearly detect $\mathrm{C} 3$ outside the interferometer primary beam (not shown in Fig. 2), while C4 is undetected.

At $225 \mathrm{GHz}$, the continuum sources C1-a and C1-b are blended into $\mathrm{C} 1$ as in the $96 \mathrm{GHz}$ image (Fig. 1), and $\mathrm{C} 2$ is clearly detected as well. As for the $284 \mathrm{GHz}$ image, C4 is undetected while $\mathrm{C} 3$ is detected but not shown because outside the primary beam, corresponding to the interferometer field of view. There is a faint emission $\sim 18^{\prime \prime}$ to the NE of C1-a and C1-b, but we decided not to consider this as a real source because it is detected at $6 \sigma$ in this image only, and quite close to the edge of the interferometer primary beam.

Because $\mathrm{C} 3$ and $\mathrm{C} 4$ are outside and on the edge of the primary beam, respectively, we decided not to discuss these sources further in the following.

Spitzer Post-Basic Calibrated Data (PBCD) at 24 and $70 \mu \mathrm{m}$ were obtained from the Spitzer Archive. The data were taken in Photometry/Super Resolution mode as part of program 20635 (R. Klein, PI). Observations at 24 microns were obtained on 7 October 2005 (AORKEY 14944768), with an integration time of $99 \mathrm{~s}$, and at 70 microns on 2 April 2006 (AORKEY 14945024) with an integration time of $76 \mathrm{~s}$ (using the Fine Scale mode). At 


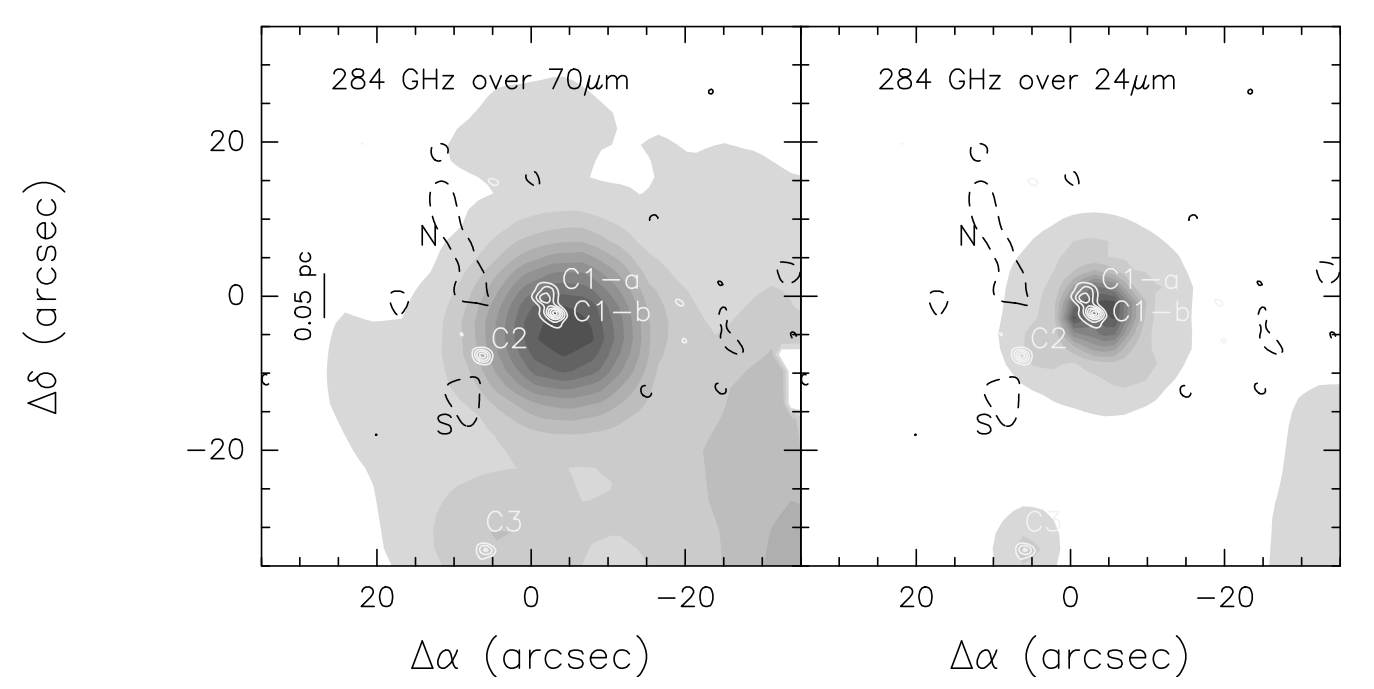

Fig. 3. Left panel: map of the $284 \mathrm{GHz}$ continuum (solid white contours) obtained with the Submillimeter Array, superimposed on the Spitzer MIPS image of I05345 at $70 \mu \mathrm{m}$, shown by the grey scale (first level and step $=10 \%$ of the maximum). The levels of the $284 \mathrm{GHz}$ image are the same as in Fig. 2. The millimeter continuum sources C1-a, C1-b and C2 are labelled as in Fig. 2. The dashed contour represents the $3 \sigma$ level of the $\mathrm{N}_{2} \mathrm{D}^{+}$(3-2) line integrated emission (see Fig. 2). Right panel: same as left panel for the $24 \mu \mathrm{m}$ Spitzer MIPS image.

present no IRAC observations have been made. The left and right panels of Fig. 3 show the Spitzer MIPS continuum images at $70 \mu \mathrm{m}$ and $24 \mu \mathrm{m}$. In both images, two peaks roughly coincident with the submillimeter continuum sources $\mathrm{C} 1$ and $\mathrm{C} 3$ are detected. At $70 \mu \mathrm{m}$, an extended emission is detected towards $\mathrm{C} 2$, $\mathrm{S}$ and the southern portion of $\mathrm{N}$, but we believe that it is just due to the low angular resolution of the map $\left(\sim 18^{\prime \prime}\right)$, which do not allow us to disentangle the contibution of the different sources. The $24 \mu \mathrm{m}$ continuum emission shows two peaks spatially coincident with the $70 \mu \mathrm{m}$ ones, but they are both more centrally peaked than at $70 \mu \mathrm{m}$, and have smaller extensions, probably due to the better angular resolution $\left(\sim 6^{\prime \prime}\right.$ at $\left.24 \mu \mathrm{m}\right)$. A faint emission is detected towards $\mathrm{C} 2$, at the edge of the main emission peak. No emission is detected towards $\mathrm{N}$ and $\mathrm{S}$ at this wavelength.

\subsection{Molecular line emission}

In Paper I we have shown and discussed the distribution of the integrated intensity of the $\mathrm{N}_{2} \mathrm{D}^{+}$and $\mathrm{N}_{2} \mathrm{H}^{+}$lines (see also Fig. 1). However, as said in Sect. 2.1, several transitions of other species have been observed and detected simultaneously as the $\mathrm{N}_{2} \mathrm{D}^{+}$ and $\mathrm{N}_{2} \mathrm{H}^{+}$ones. In this section, we complete the analysis started in Paper I and show a full report of the observations obtained.

\subsubsection{Integrated emission map of the optically thin component of the $\mathrm{N}_{2} \mathrm{H}^{+}(1-0)$ line}

The integrated intensity of the main group of hyperfine components of the $\mathrm{N}_{2} \mathrm{H}^{+}(1-0)$ line is shown in Fig. 1. As already discussed in Sect. 1, the emission is extended and presents several bumps which are not resolved into separate emission peaks. This may be the real shape of the emission in this tracer, or it can be due to other reasons, such as an insufficient angular resolution, or opacity effects. To better understand this, in Fig. 4 we show the distribution of the integrated intensity of the hyperfine component $F_{1} F=10 \rightarrow 11$ only, which is well separated from the other components and it is expected to be optically thin: from the fitting procedure described in Sect. 2.4, we obtain an optical depth for this component of $\sim 0.4 \div 0.7$. Figure 4 shows that the

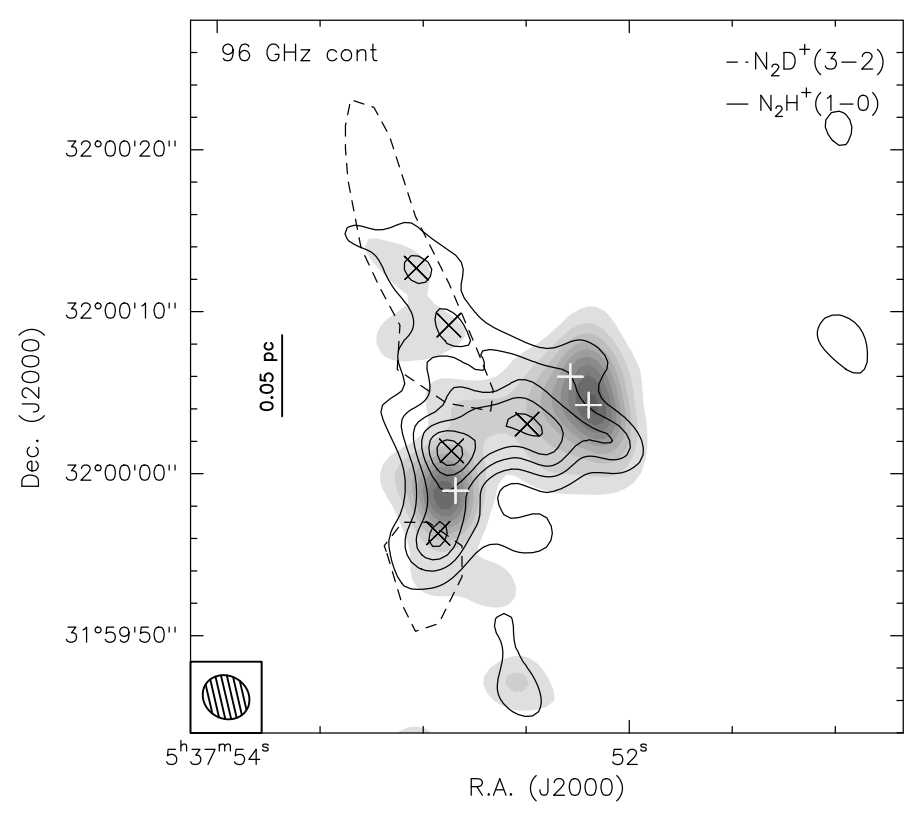

Fig. 4. Integrated intensity of the hyperfine component $F_{1} F=$ $10 \rightarrow 11$ of the $\mathrm{N}_{2} \mathrm{H}^{+}$(1-0) line (solid contours), superimposed on the $96 \mathrm{GHz}$ continuum (grey-scale). The contours start from the $3 \sigma$ $\left(0.018 \mathrm{Jy} \mathrm{beam}^{-1}\right)$, and are in steps of $3 \sigma$. The grey-scale is the same as in Fig. 2. The white crosses correspond to the three peaks detected in the $284 \mathrm{GHz}$ continuum image, and the black crosses mark the position of the $\mathrm{N}_{2} \mathrm{H}^{+}$emission peaks. The dashed contrours represent the $3 \sigma$ level of the $\mathrm{N}_{2} \mathrm{D}^{+}$(3-2) integrated emission. The ellipse in the bottom-left corner is the synthesised beam.

extension of the integrated intensity of this component is similar to that shown in Fig. 1, but in this case we do detect five emission peaks. This suggests that, since the angular resolution is the same, the lack of well separated intensity peaks in Fig. 1 is probably due to optical depth effects.

The main emission peak is located $\sim 4^{\prime \prime}$ north of $\mathrm{C} 2$, while the others are located about $\sim 5^{\prime \prime}$ east of $\mathrm{C} 1$, and $\sim 5^{\prime \prime}$ south of $\mathrm{C} 2$, respectively, and two fainter peaks (detected at $\sim 6 \sigma$ ) are seen towards core $\mathrm{N}$. None of the millimeter sources coincide with 
Table 2. Line parameters for calculations of the ${ }^{12} \mathrm{CO}(2-1)$ outflow.

\begin{tabular}{cccccc}
\hline \hline $\begin{array}{c}\Delta V_{\mathrm{b}} \\
\left(\mathrm{km} \mathrm{s}^{-1}\right)\end{array}$ & $\begin{array}{c}\Delta V_{\mathrm{r}} \\
\left(\mathrm{km} \mathrm{s}^{-1}\right)\end{array}$ & $\begin{array}{c}\left.\int T \mathrm{~d} V \text { (blue }\right) \\
\left(\mathrm{K} \mathrm{km} \mathrm{s}^{-1}\right)\end{array}$ & $\begin{array}{c}\int T \mathrm{~d} V(\mathrm{red}) \\
\left(\mathrm{K} \mathrm{km} \mathrm{s}^{-1}\right)\end{array}$ & $\begin{array}{c}V_{\max _{\mathrm{b}}} \\
\left(\mathrm{km} \mathrm{s}^{-1}\right)\end{array}$ & $\begin{array}{c}V_{\max _{\mathrm{r}}} \\
\left(\mathrm{km} \mathrm{s}^{-1}\right)\end{array}$ \\
\hline$-46.4,-26$ & $-9.2,6.4$ & 1329.3 & 1663.4 & 28 & 24.8 \\
\hline
\end{tabular}

the five $\mathrm{N}_{2} \mathrm{H}^{+}$emission peaks. These results indicate that the source can host other dense cores which are not detected in the millimeter continuum observations, probably because they are too cold.

\subsubsection{Integrated emission map of the ${ }^{12} \mathrm{CO}(2-1)$ line wings}

Single-dish observations of the source in the ${ }^{12} \mathrm{CO}(2-1)$ line have been performed by Zhang et al. (2005), with a 29" angular resolution. They detected a massive outflow in the non-Gaussian line wings, the orientation of which is approximately in the WE direction, but the angular resolution was not sufficient to detect either the outflow center and the detailed morphology of the outflow itself. The observations reported in this work significantly improve the angular resolution of the previous ones, allowing us to better determine the outflow shape and identify its origin.

In Fig. 5, we show the map of the integrated intensity in the ${ }^{12} \mathrm{CO}(2-1)$ line wings, derived from channel maps obtained combining SMA and NRAO data, as described in Sect. 2.3. The blue- and red-shifted emissions have been averaged in the velocity intervals $(-46.4,-26) \mathrm{km} \mathrm{s}^{-1}$ and $(-9.2,6.4) \mathrm{km} \mathrm{s}^{-1}$, respectively. The outflow axis is predominantly oriented in the WE direction, with redshifted gas in the east and blueshifted gas in the west. The lobes are clearly separated and have approximately a biconical shape. The outflow center is near the position of the continuum source $\mathrm{C} 1$, and the exciting source can be either $\mathrm{C} 1$-a and $\mathrm{C} 1-\mathrm{b}$. The source coincident with the most western peak of the $\mathrm{N}_{2} \mathrm{H}^{+}$(1-0) line integrated emission can also contribute to the observed emission, but is less likely to contribute than the continuum sources, due to the fact that there are no other signs of protostellar activity associated with it. The blueshifted gas shows a fainter secondary peak to the north of the map. From geometrical considerations, this northern blue-shifted emission might be driven by a source within $\mathrm{N}$, rather than $\mathrm{C} 1-\mathrm{a}$ or $\mathrm{C} 1-\mathrm{b}$. However, as we will further discuss later, the sources embedded within $\mathrm{N}$ are probably in the pre-stellar phase, i.e. prior to the main accretion phase in which the outflow is expected to form, so that this solution seems to us very unlikely.

The outflow length, from end-to-end, is approximately $35^{\prime \prime}$ (if we do not consider the secondary peaks of the blueshifted emission), corresponding to $\sim 0.28 \mathrm{pc}$ at a distance of $1.8 \mathrm{kpc}$. The semi-opening angle is between about 30 and $40^{\circ}$, and the spatial separation of the lobes suggests that the inclination angle with respect to the line of sight is likely to be very close to the plane of the sky (see also Cabrit \& Bertout 1986).

In Table 2 we give the line parameters for the calculation of the outflow properties, namely: the velocity range of the blueand red-wings, $\Delta V_{\mathrm{b}}$ and $\Delta V_{\mathrm{r}}$ (Cols. 1 and 2, respectively), the integrated intensity of ${ }^{12} \mathrm{CO}(2-1)$ in the wings, $\int T \mathrm{~d} V$ (blue) and (red) (Cols. 3 and 4; $T$ is the main beam brigthness temperature), and the maximum velocity of the wings, $V_{\max _{\mathrm{b}}}$ and $V_{\max _{\mathrm{r}}}$ (Cols. 5 and 6), defined as the difference between the maximum velocity of the blue- and red-wings and the systemic velocity (i.e. $-18.4 \mathrm{~km} \mathrm{~s}^{-1}$ ). The values listed in Cols. 3 and 4 are averaged values over the blue and the red lobes, respectively.

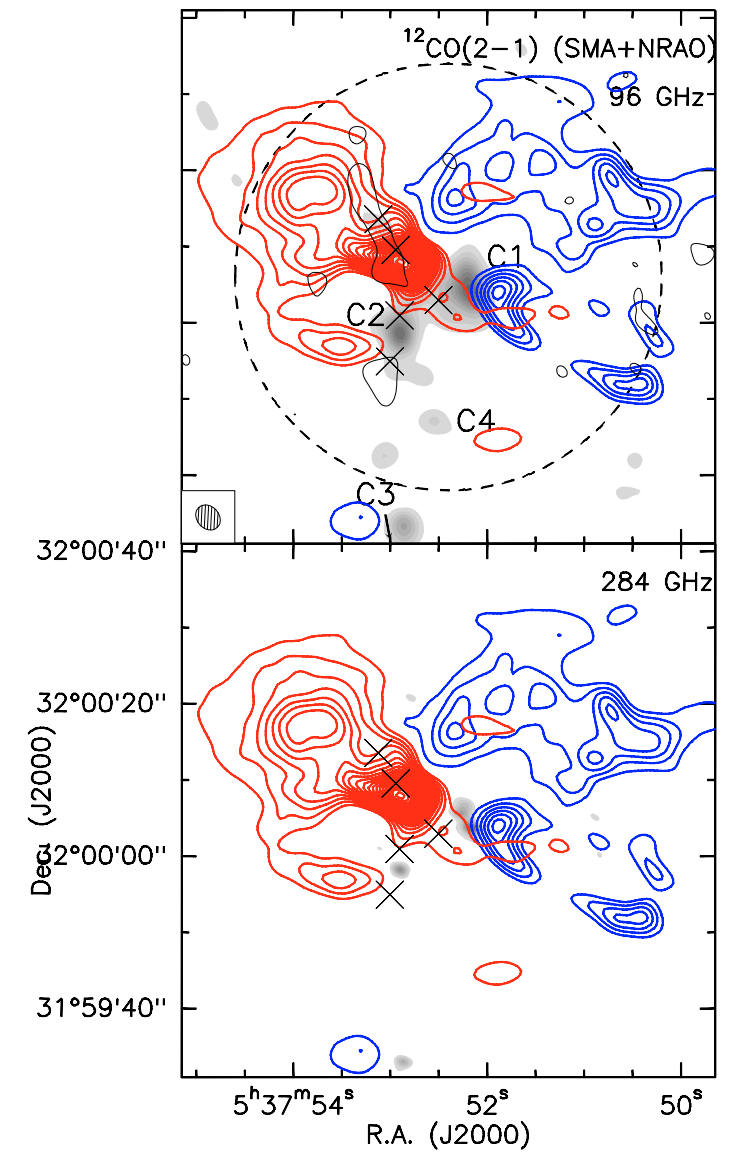

Fig. 5. Top panel: red- and blue-shifted integrated emission of the ${ }^{12} \mathrm{CO}(2-1)$ line (combined SMA + NRAO data), superimposed on the $96 \mathrm{GHz}$ continuum map observed with the PdBI (grey-scale). Both red and blue contours start from $0.25 \mathrm{Jy}_{\text {beam }}{ }^{-1}(\sim 3 \sigma \mathrm{rms})$, and are in steps of $0.25 \mathrm{Jy} \mathrm{beam}^{-1}$. The solid black contours represent the $3 \sigma$ level of the $\mathrm{N}_{2} \mathrm{D}^{+}$(3-2) line integrated emission, and indicate the location of the deuterated cores $\mathrm{N}$ and $\mathrm{S}$ (see also Fig. 1). The crosses correspond to the five $\mathrm{N}_{2} \mathrm{H}^{+}$(1-0) emission peaks shown in Fig. 4. The dashed circle represents the SMA primary beam at the frequency of the ${ }^{12} \mathrm{CO}(2-1)$ line. The beam of the combined ${ }^{12} \mathrm{CO}$ map is shown in the bottom left corner. Bottom panel: same as top panel but the grey-scale corresponds to the continuum at $284 \mathrm{GHz}$ observed with SMA. The $3 \sigma$ level of the $\mathrm{N}_{2} \mathrm{D}^{+}(3-2)$ emission and the interferometer primary beam are not shown here.

\subsubsection{Integrated intensity of other lines}

In Fig. 6 we show the distribution of the integrated intensity of all the detected transitions listed in Table 1, averaged over the channels with signal, except for ${ }^{12} \mathrm{CO}(2-1)$ that has been already discussed.

The integrated intensity of the ${ }^{13} \mathrm{CO}(2-1)$ line peaks at the position of $\mathrm{C} 1$, but it shows an elongated shape towards the north-east, suggesting that some of the emission comes from the outflow. On the other hand, the $\mathrm{C}^{18} \mathrm{O}(2-1)$ line emission is less extended and it seems to arise from a common gaseous envelope in which $\mathrm{C} 1-\mathrm{a}$ and $\mathrm{C} 1-\mathrm{b}$ are embedded. No significant emission 


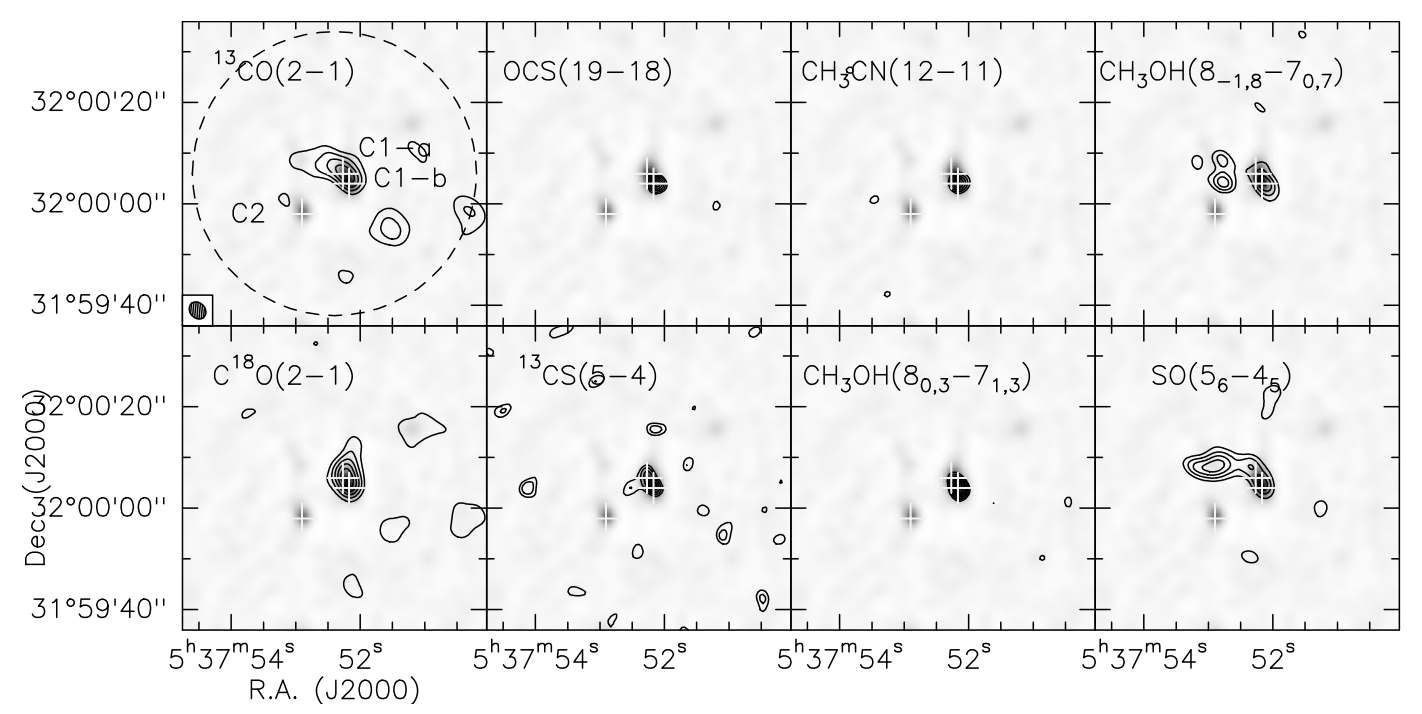

Fig. 6. Integrated maps of all the molecular lines listed in Table 1, detected towards I05345 with the SMA. For all transitions, the emission has been averaged over all the channels with signal. In each panel, the transition is indicated in the top left corner, the grey scale represents the $94 \mathrm{GHz}$ continuum, and the position of the mm cores is indicated by the white crosses. For ${ }^{13} \mathrm{CO}, \mathrm{C}^{18} \mathrm{O}, \mathrm{CH}_{3} \mathrm{OH}\left(8_{-1,8}-7_{0,7}\right)$ and $\mathrm{SO}$, the contours start from the $3 \sigma$ level of the averaged map, and are in steps of $3 \sigma$, while for the other lines are in step of $1 \sigma$. In the top left panel, where the ${ }^{13} \mathrm{CO}(2-1)$ line is shown, the ellipse in the bottom left corner indicates the synthesised beam, and the dashed circle corresponds to the interferometer primary beam. The other lines have the same primary beam and similar synthesised beams.

of this line is detected towards the ${ }^{12} \mathrm{CO}$ outflow lobes. A similar distribution is seen in the integrated emission of ${ }^{13} \mathrm{CS}(5-4)$ and, slightly less, in $\mathrm{CH}_{3} \mathrm{OH}\left(8_{0,3}-7_{1,3}\right)$, showing that these transitions trace the same gas.

The integrated intensity of the two high excitation lines OCS (19-18) and $\mathrm{CH}_{3} \mathrm{CN}(12-11)$ clearly shows the presence of a compact hot core centered on source C1-b. In both tracers, the hot core is unresolved, so that its diameter has an upper limit of approximately $3^{\prime \prime}$, i.e. $\sim 0.026 \mathrm{pc}$ at the given distance. The hot-core nature of this condensation is confirmed by the gas temperature of $\sim 200 \mathrm{~K}$ that we will derive in Sect. 4.1.

For the $\mathrm{CH}_{3} \mathrm{OH}\left(8_{-1,8}-7_{0,7}\right)$ and SO $\left(5_{6}-4_{5}\right)$ line, the emission looks clumpy, with a main spot corresponding to the position of $\mathrm{C} 1$, and another prominent spot coincident with the redshifted ${ }^{12} \mathrm{CO}$ emission, and several fainter spots north and west of $\mathrm{C} 1$ which do not coincide with any of the other tracers observed. The strong emission of SO $\left(5_{6}-4_{5}\right)$ in the red lobe of the ${ }^{12} \mathrm{CO}$ outflow is consistent with previous studies, in which it has been found that SO abundance is enhanced in outflows (Bachiller et al. 2001; Viti et al. 2003), and it has been clearly detected in other massive outflows studied at high-angular reoslution (see e.g. the case study of AFGL 5142, Zhang et al. 2007).

On the other hand, the $\mathrm{CH}_{3} \mathrm{OH}\left(8_{-1,8}-7_{0,7}\right)$ line is believed to be a Class I methanol maser line, and it has been detected at low-angular resolution in several high-mass star forming regions (see e.g. Slysh et al. 2002). Recently, the SMA has imaged several masers in this line towards the source DR21 $(\mathrm{OH})$ (Bourke, priv. comm.). Class I methanol masers are often located offset from the position of hot cores or HII regions, where Class II and other masers, such as those of water and $\mathrm{OH}$, are found. They are believed to be collisionally excited, probably where a powerful outflow impacts the surrounding quiescent material (see e.g. Sandell et al. 2003). However, high angular resolution observations have shown that in some cases they can be found in close proximity to HII regions and water masers (Kurtz et al. 2004). The map shown in Fig. 6 clearly shows that the $\left(8_{-1,8}-7_{0,7}\right)$ line comes from both the hot core and the red lobe of the outflow, indicating that it is generated by shocked gas, and the spatial extent is similar to that of $\mathrm{SO}$, even though the SO emission is more prominent in the outflow than that of $\mathrm{CH}_{3} \mathrm{OH}$. To better understand the nature of the $\mathrm{CH}_{3} \mathrm{OH}\left(8_{-1,8}-7_{0,7}\right)$ line, in Fig. 7 we show the spectra of this line towards two positions at an offset of $\left(-3^{\prime \prime},-2^{\prime \prime}\right)$ and $\left(+5^{\prime \prime},-1.5^{\prime \prime}\right)$ from the map centre. These two positions correspond to the main emission peaks of the averaged map, and roughly coincide with $\mathrm{C} 1$ and with the inner part of the red lobe of the ${ }^{12} \mathrm{CO}$ outflow, respectively (see Fig. 6). We clearly see that the line in the outflow is much narrower than the one observed towards core $\mathrm{C} 1\left(\sim 5 \mathrm{~km} \mathrm{~s}^{-1}\right.$ and $\sim 1 \mathrm{~km} \mathrm{~s}^{-1}$, respectively). The broad line seen towards $\mathrm{C} 1$ is likely thermal, while the narrow one observed towards the red lobe of the ${ }^{12} \mathrm{CO}$ outflow could come from a single maser spot, even though the flux density is not very high ( $\sim .08 \mathrm{Jy})$ and could also be thermal as well.

Finally, to better highlight the emission of ${ }^{13} \mathrm{CO}$ and $\mathrm{SO}$ in the outflow, in Fig. 8 we show the integrated intensity maps of the blue- and red-shifted emission of the ${ }^{13} \mathrm{CO}(2-1)$ and SO $\left(5_{6}-4_{5}\right)$ lines. The blue-shifted emission of ${ }^{13} \mathrm{CO}$ seems to trace the cavity created by the blue lobe of the ${ }^{12} \mathrm{CO}$ outflow, while the red-shifted emission follows the inner part of the ${ }^{12} \mathrm{CO}$ red lobe (see upper panel of Fig. 8). For SO $\left(5_{6}-4_{5}\right)$, we find a similar result for the red-shifted emission, while the blue-shifted emission of this line does not follow that of ${ }^{12} \mathrm{CO}$, but it arises mostly from core $\mathrm{C} 1$ and from the ${ }^{12} \mathrm{CO}$ red lobe. This strange feature could be explained by the presence of another outflow not detected in ${ }^{12} \mathrm{CO}$, centered roughly in between core $\mathrm{N}$ and core $\mathrm{C} 1$. A northern bump in the blue-shifted emission is also detected, located at the position of the northern blueshifted emission seen in Fig. 5.

\section{Derivation of the physical parameters}

\subsection{Temperature of core $\mathrm{C1-b}$ from $\mathrm{CH}_{3} \mathrm{CN}$}

Figure 9 shows the $\mathrm{CH}_{3} \mathrm{CN}(12-11)$ spectrum at the peak position of the emission map shown in Fig. $6 . \mathrm{CH}_{3} \mathrm{CN}$ is a 


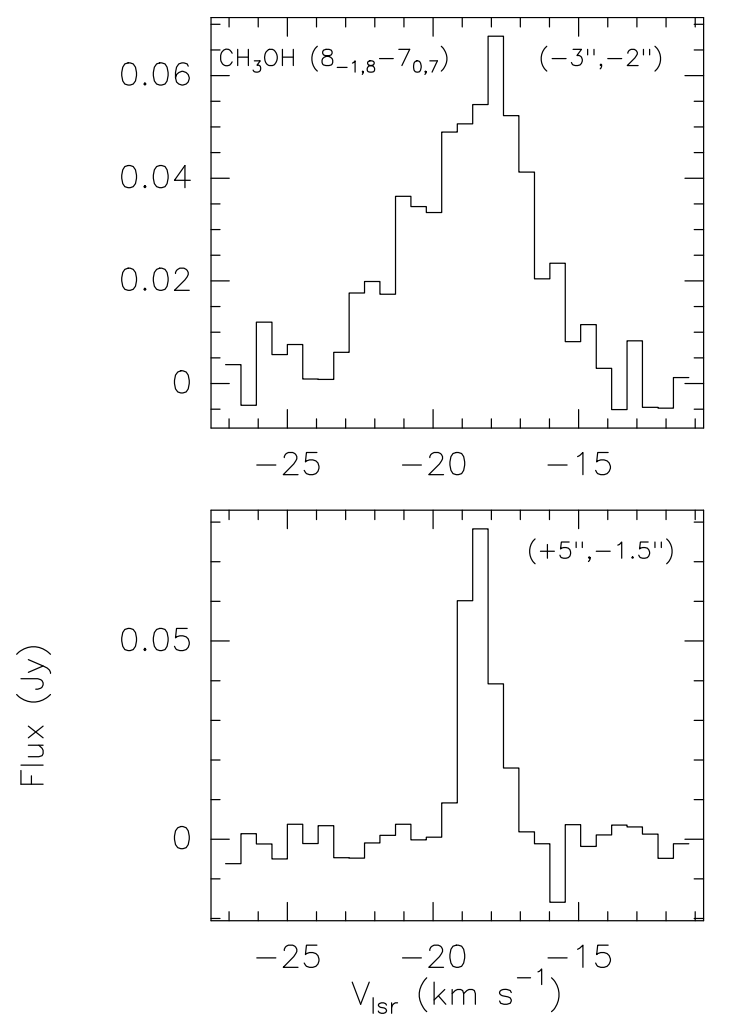

Fig. 7. Spectra of the $\mathrm{CH}_{3} \mathrm{OH}\left(8_{-1,8}-7_{0,7}\right)$ line obtained towards the positions of the two main emission peaks seen in the averaged map of this line (Fig. 6). The offsets (in arcsec) are indicated in the top-right corner, and correspond to the peak position of $\mathrm{C} 1$ and the "base" of the red lobe of the ${ }^{12} \mathrm{CO}$ outflow.

symmetric-top molecule whose rotational levels are described by two quantum numbers: $J$, associated with the total angular momentum, and $K$, its projection on the symmetry axis. Such a structure entails that for each radiative transition, $J+1 \rightarrow J$, lines with $K \leq J$ can be seen (for a detailed description see Townes $\&$ Schawlow 1975). In our observations the bandwidth covers up to the $K=7$ component for the (12-11) transition. However, we detected only lines up to $K=4$, for which the energy of the upper level is $184 \mathrm{~K}$. In order to compute the line parameters, we performed Gaussian fits to the observed spectrum assuming that all the $K$ components arise from the same gas. Hence, they have the same LSR velocity and line width. We fixed the line separation in the spectrum to the laboratory value and derive a line width by fitting the spectrum of all the $K$ components. Then, under the assumption of LTE conditions, we compute the $\mathrm{CH}_{3} \mathrm{CN}$ spectrum using the gas temperature, the $\mathrm{CH}_{3} \mathrm{CN}$ density, the source size as input parameters, and a line width fixed to the observed value (Qiu 2009, private communication). This approach does not assume optically thin emission in $\mathrm{CH}_{3} \mathrm{CN}$, as does in the Boltzmann analysis (see e.g. Kuiper et al. 1984; Bergin et al. 1994). The best fit to the $\mathrm{CH}_{3} \mathrm{CN}$ spectrum shown in Fig. 9 yields a range of kinetic temperatures from 150-250 K. These values are comparable to typical temperatures found in hot molecular cores surrounding a newly formed massive star (see e.g. Kurtz et al. 2000).

\subsection{Physical parameters from dust emission}

The physical parameters derived from the millimeter continuum images presented in Sect. 3.1 are listed in Table 3: the position of the emission peak, in RA (J2000) and Dec (J2000), of C1

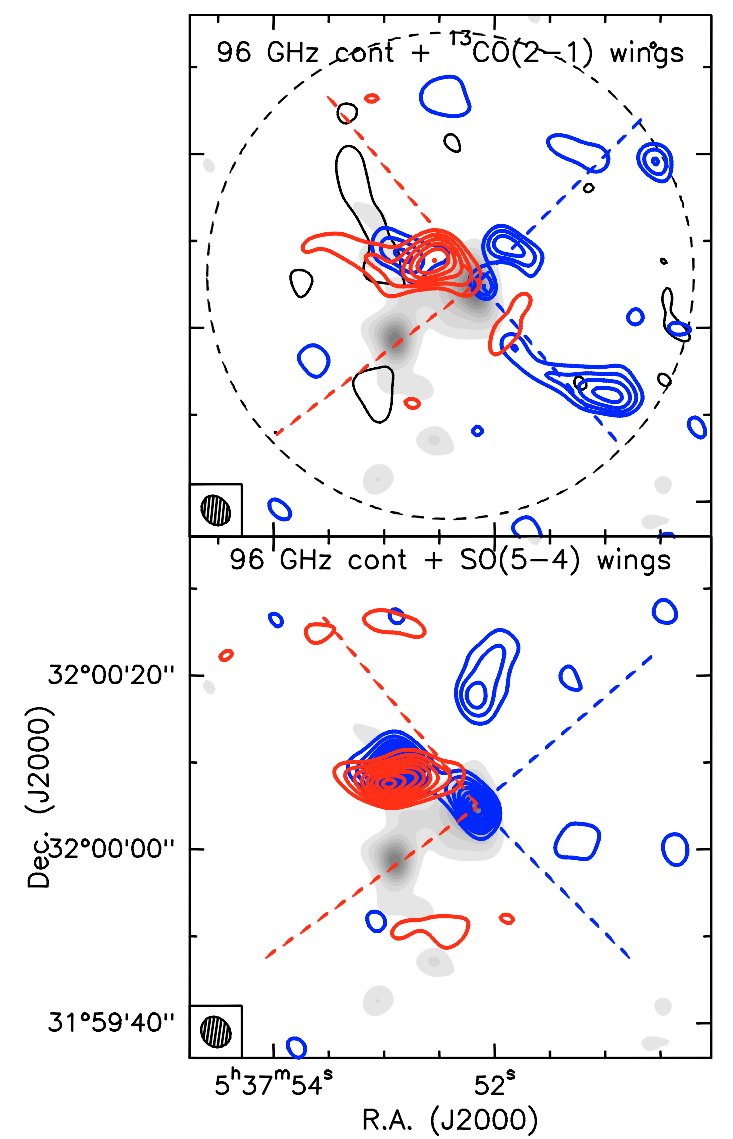

Fig. 8. Top panel: red- and blue-shifted integrated emission of the ${ }^{13} \mathrm{CO}(2-1)$ line observed with SMA, superimposed on the $96 \mathrm{GHz}$ continuum map observed with the PdBI (grey-scale). For this latter we use the same contours as in Fig. 5. The integration ranges in velocity are $(-15.88 ;-8.14)$ and $(-23.06 ;-20.85) \mathrm{km} \mathrm{s}^{-1}$. The red contours start from $0.16 \mathrm{Jy} \mathrm{beam}^{-1}(\sim 3 \sigma \mathrm{rms})$, and are in steps of $0.16 \mathrm{Jy}^{\mathrm{beam}}{ }^{-1}$. The blue contours start from $0.24 \mathrm{Jy}_{\text {beam }}^{-1}(\sim 3 \sigma \mathrm{rms})$, and are in steps of $0.16 \mathrm{Jy} \mathrm{beam}^{-1}$. The solid black contours represent the $3 \sigma$ level of the $\mathrm{N}_{2} \mathrm{D}^{+}$(3-2) line integrated emission as in Fig. 5. The dashed circle corresponds to the SMA primary beam at the frequency of the ${ }^{13} \mathrm{CO}(2-$ 1) line. The red- and blue-dashed lines indicate the opening angle of the ${ }^{12} \mathrm{CO}$ outflow lobes as in Fig. 5 (excluding the northern blue bump). The SMA synthesised beam is shown in the bottom left corner. Bottom panel: same as top panel for the SO $\left(5_{6}-4_{5}\right)$ line. The integration ranges in velocity are $(-23.5 ;-20.2)$ and $(-14 ;-3) \mathrm{km} \mathrm{s}^{-1}$. For both red- and blue-shifted emission, both the first contour and the step is the $3 \sigma$ of the averaged map ( 0.06 and $0.09 \mathrm{Jy}_{\text {beam }}{ }^{-1}$, respectively). The $3 \sigma$ level of the $\mathrm{N}_{2} \mathrm{D}^{+}(3-2)$ emission and the interferometer primary beam are not shown here.

(resolved into $\mathrm{C} 1-\mathrm{a}$ and $\mathrm{C} 1-\mathrm{b}$ at $284 \mathrm{GHz}$ ) and $\mathrm{C} 2$ are listed in Cols. 2 and 3, respectively; the deconvolved angular $(\theta)$ and linear $(D)$ diameters are given in Cols. 4 and 5. Assuming that the emission has a Gaussian profile, we derived the angular diameter of the sources deconvolving the observed FWHM with a Gaussian corresponding to the synthesised beam of the interferometer. The observed FWHM was measured as the geometrical mean of major and minor axes of the contour at half of the intensity peak. The linear diameters were computed using a source distance of $1.8 \mathrm{kpc}$ (Zhang et al. 2005).

In Cols. 6 of Table 3 we also give the flux density, $F_{v}$, derived integrating over the $3 \sigma$ level in each core. Column 7 shows the gas masses obtained from $F_{v}$ : assuming constant gas-to-dust 
Table 3. Peak position, angular and linear diameter, integrated flux density, mass, $\mathrm{H}_{2}$ volume and column density of the millimeter condensations $\mathrm{C} 1$ (resolved into $\mathrm{C} 1-\mathrm{a}$ and $\mathrm{C} 1 \mathrm{-b}$ in the $284 \mathrm{GHz}$ image) and $\mathrm{C} 2$. The masses are computed for $\beta=2$, and assuming $T=30 \mathrm{~K}$. The $\mathrm{H}_{2}$ volume and column densities are calculated assuming a spherical source with diameter equal to the deconvolved $3 \sigma$ level.

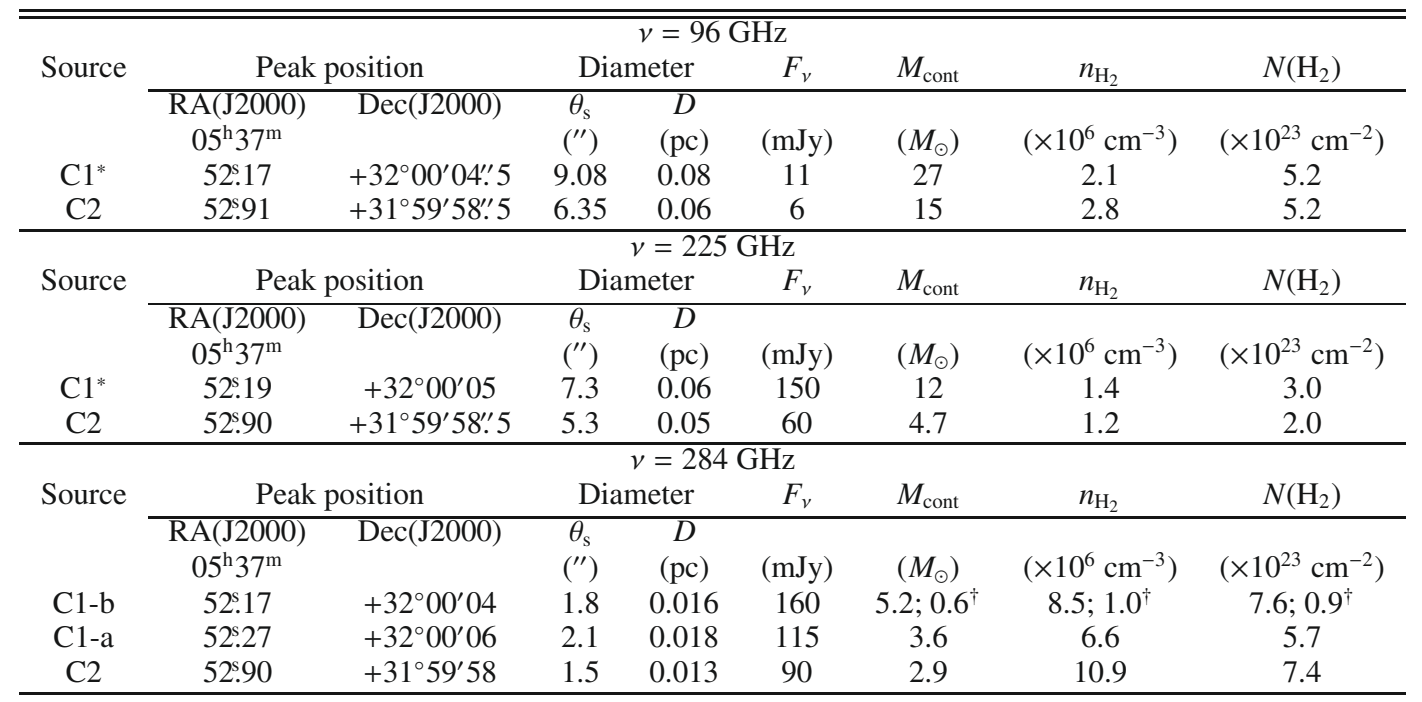

${ }^{*} \mathrm{C} 1-\mathrm{a}+\mathrm{C} 1-\mathrm{b}$

${ }^{\dagger}$ Computed from the $\mathrm{CH}_{3} \mathrm{CN}$ temperature of $200 \mathrm{~K}$.

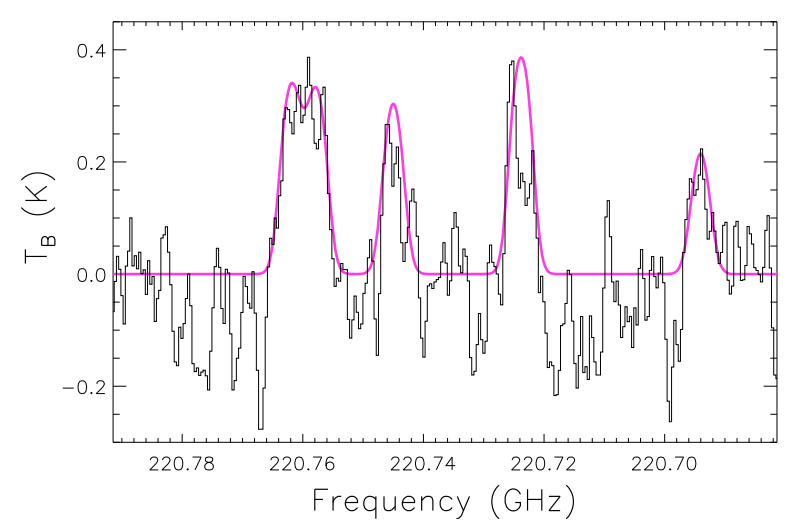

Fig. 9. Spectrum of the $\mathrm{CH}_{3} \mathrm{CN}(12-11)$ line at the peak position, obtained with the SMA. The red line represents the best fit to the components $K=0$ to $K=4$, performed as explained in Sect. 4.1.

ratio, optically thin and isothermal conditions, the total gas+dust mass is given by:

$M_{\mathrm{cont}}=\frac{F_{v} d^{2}}{k_{v} B_{v}(T)}$.

In Eq. (1), $d$ is the distance, $k_{v}$ is the dust opacity coefficient, derived according to $k_{v}=k_{\nu_{0}}\left(v / v_{0}\right)^{\beta}$ (where $v_{0}=230 \mathrm{GHz}$ and $k_{v_{0}}=0.005 \mathrm{~cm}^{2} \mathrm{~g}^{-1}$, which implies a gas-to-dust ratio of 100 , Kramer et al. 1998), and $B_{v}(T)$ is the Planck function calculated at the dust temperature $T$. For this latter, since we do not have a direct estimate (except for C1-b), we have assumed a "reasonable" value of $30 \mathrm{~K}$ (see e.g. Molinari et al. 2000). For C1-b, for which we also have a direct temperature measurement from $\mathrm{CH}_{3} \mathrm{CN}$ (see Sect. 4.1), we give two estimates: one assuming $T=30 \mathrm{~K}$ and the other assuming the value derived from the $\mathrm{CH}_{3} \mathrm{CN}$ spectrum, i.e. $T=200 \mathrm{~K}$. For the dust grain emissivity index, we assumed $\beta=2$ (see e.g. Hill et al. 2006). From the $3 \sigma$ level in the continuum images, we have also derived that we are sensitive to point-like sources with masses of $0.6,0.2$ and $0.01 M_{\odot}$ at 284, 225 and $96 \mathrm{GHz}$, respectively (assuming $T=30 \mathrm{~K})$. We also estimate a column density sensitivity of $\sim 7 \times 10^{22}, 1.9 \times 10^{22}$ and $1.4 \times 10^{21} \mathrm{~cm}^{-2}$ at the three frequencies mentioned above.

In Cols. 8 and 9 of Table 3 , we also list the average $\mathrm{H}_{2}$ volume and column densities. The $\mathrm{H}_{2}$ average volume densities were computed assuming spherical cores, then the column densities were derived by multiplying the volume densities for the core diameters. We have used as angular diameters those obtained deconvolving the $3 \sigma$ levels in the continuum images, and not those listed in Table 3 because the continuum fluxes were derived integrating the emission within the $3 \sigma$ contours. All volume densities are of the order of $10^{6} \mathrm{~cm}^{-3}$, while the column densities are of the order of $10^{23} \mathrm{~cm}^{-2}$, which are typical values found in cores associated with intermediate-/high-mass forming stars, and are higher than the critical value of $1 \mathrm{~g} \mathrm{~cm}^{-2}$ required to form a massive star at the core centre (Krumholz et al. 2008), assuming that the gas is totally made up of $\mathrm{H}_{2}$.

The mass of $\mathrm{C} 2$, estimated to be $11.8,4.7$ and $\sim 2.9 M_{\odot}$ at 96,225 and $284 \mathrm{GHz}$, indicates that the core probably hosts an intermediate-mass object. The mass of $\mathrm{C} 1$ is 27 and $11.8 M_{\odot}$ from the 96 and $225 \mathrm{GHz}$ continuum emission, and when it is resolved in two components, $\mathrm{C} 1-\mathrm{a}$ and $\mathrm{C} 1-\mathrm{b}$, in the $284 \mathrm{GHz}$ image, the masses of the components are 3.8 and 5.2 $M_{\odot}$, respectively. These values are typical of cores associated with intermediate-mass protostellar objects (see e.g. Beltràn et al. 2008). For C1-b, adopting the temperature of $200 \mathrm{~K}$ from $\mathrm{CH}_{3} \mathrm{CN}$, we even obtain $0.6 M_{\odot}$. However, as we will discuss in Sect. 5.1, the source embedded in core C1-b is likely an early-B newly formed star. Therefore, the gas mass derived from the millimeter continuum represents for this source the mass of the circumstellar material only, and not that of the central star.

Additionally, for all sources these mass estimates, especially those derived from the $284 \mathrm{GHz}$ continuum, are very uncertain because they are affected by several problems. First, both the assumed dust temperature and $\beta$ are very uncertain: $\beta$ is expected to be between 1 and 2 in high-mass star forming regions (see e.g. Hill et al. 2006, and references therein), while the dust 
Table 4. Physical parameters derived in Paper I for condensations $\mathrm{N}$ and $\mathrm{S}$ from $\mathrm{N}_{2} \mathrm{H}^{+}$and $\mathrm{N}_{2} \mathrm{D}^{+}$.

\begin{tabular}{cccccc}
\hline \hline Source & $\begin{array}{c}N\left(\mathrm{~N}_{2} \mathrm{H}^{+}\right) \\
\left(\times 10^{13} \mathrm{~cm}^{-2}\right)\end{array}$ & $\begin{array}{c}N\left(\mathrm{~N}_{2} \mathrm{D}^{+}\right) \\
\left(\times 10^{12} \mathrm{~cm}^{-2}\right)\end{array}$ & $D_{\text {frac }}$ & $\begin{array}{c}L \\
(\mathrm{pc})\end{array}$ & $\begin{array}{c}M_{\mathrm{N}_{2} \mathrm{H}^{+}} \\
\left(M_{\odot}\right)\end{array}$ \\
\hline $\mathrm{N}$ & $1.9(0.6)$ & $2.1(0.3)$ & $0.11(0.04)$ & 0.09 & 8.7 \\
$\mathrm{~S}$ & $1.5(0.5)$ & $1.6(0.3)$ & $0.11(0.04)$ & 0.05 & 2.5 \\
\hline
\end{tabular}

temperature can vary considerably from a source to another (see e.g. Molinari et al. 2000; Sridharan et al. 2002). For example, assuming the gas temperature derived from $\mathrm{NH}_{3}$ observations, that is $T=17 \mathrm{~K}$ (Jijina et al. 1999), with $\beta=2$ we get 7.9 and $10.9 M_{\odot}$ for $\mathrm{C} 1-\mathrm{a}$ and $\mathrm{C} 1-\mathrm{b}$, respectively, and 8.8 and 12.2 assuming $T=17 \mathrm{~K}$ and $\beta=1.5$ (Mathis $\&$ Wiffen 1989). Second, the $284 \mathrm{GHz}$ image is more affected than the others by the problem of the missing flux, so that the core masses derived from this image are representative of the circumstellar material very close to the central object only, while those from the 225 and $96 \mathrm{GHz}$ continuum are more representative of the whole envelope. To estimate the amount of flux filtered out beacuse of missing short spacing information, we have compared the flux measured with the single-dish SCUBA map at $850 \mu \mathrm{m}$ (Fontani et al. 2006) to that measured in the $284 \mathrm{GHz}$ map: the peak flux of the singledish map is $\sim 2.0 \mathrm{Jy} \mathrm{beam}^{-1}$. Assuming a spectral index of 4, the peak intensity at $1 \mathrm{~mm}$ (i.e. $284 \mathrm{GHz}$ ) is $1.04 \mathrm{Jy} \mathrm{beam}^{-1}$. After smoothing the interferometric map at the SCUBA angular resolution of $14^{\prime \prime}$, the flux density in the area corresponding to the SCUBA beam is $0.11 \mathrm{Jy}^{\text {beam }}{ }^{-1}$, which means that we are recovering only $\sim 11 \%$ of the total flux. Therefore, the masses derived from the $284 \mathrm{GHz}$ continuum maps have to be considered as lower limits for the circumstellar masses. On the other hand, at $96 \mathrm{GHz}$, the expected flux is $0.011 \mathrm{Jy} \mathrm{beam}^{-1}$ while the value measured from the PdBI map is $0.0075 \mathrm{Jy} \mathrm{beam}^{-1}$, so that at this frequency we recover of $68 \%$ of the total flux.

\subsection{Physical parameters from $\mathrm{N}_{2} \mathrm{H}^{+}$and $\mathrm{N}_{2} \mathrm{D}^{+}$}

\subsubsection{Deuterium fractionation}

For completeness, in Table 4, we give the physical parameters derived in Paper I for condensations $\mathrm{N}$ and $\mathrm{S}$ from $\mathrm{N}_{2} \mathrm{H}^{+}$and $\mathrm{N}_{2} \mathrm{D}^{+}$: the $\mathrm{N}_{2} \mathrm{H}^{+}$and $\mathrm{N}_{2} \mathrm{D}^{+}$total column densities $\left(N\left(\mathrm{~N}_{2} \mathrm{H}^{+}\right)\right.$ and $N\left(\mathrm{~N}_{2} \mathrm{D}^{+}\right)$, Cols. 2 and 3$)$; the deuterium fractionation, $\left(D_{\text {frac }}\right.$, Col. 4); the linear diameter ( $L$, Col. 5$)$; the mass derived from $N\left(\mathrm{~N}_{2} \mathrm{H}^{+}\right)\left(M_{\mathrm{N}_{2} \mathrm{H}^{+}}\right.$, Col. 6). The uncertainties computed following the standard propagation of the errors are given between parentheses. For more details on the methods used as well as in the assumptions made, see Sect. 3.1 of Paper I. As already discussed, in both condensations, $D_{\text {frac }}$ is 0.11 , which is comparable to the values of $D_{\text {frac }}$ found in low-mass pre-stellar cores by Crapsi et al. (2005), following the same method. These values are also comparable to those derived by Pillai et al. (2007) in infrared-dark clouds from deuterated ammonia. However, their observations are related to the very cold, pc-scale molecular envelope, and not to compact sub-pc scale cores.

We could not derive the column densities of $\mathrm{N}$ and $\mathrm{S}$ from the $\mathrm{N}_{2} \mathrm{H}^{+}$(3-2) line because this is undetected towards the two molecular condensations, as shown in Fig. 1 of Paper I. Such a different distribution of the integrated intensity with respect to that of the $\mathrm{N}_{2} \mathrm{H}^{+}(1-0)$ line is probably due to the extended emission being filtered out differently by the two interferometers. To compute the amount of the missing flux, we have compared our interferometric spectra with the single-dish spectra obtained
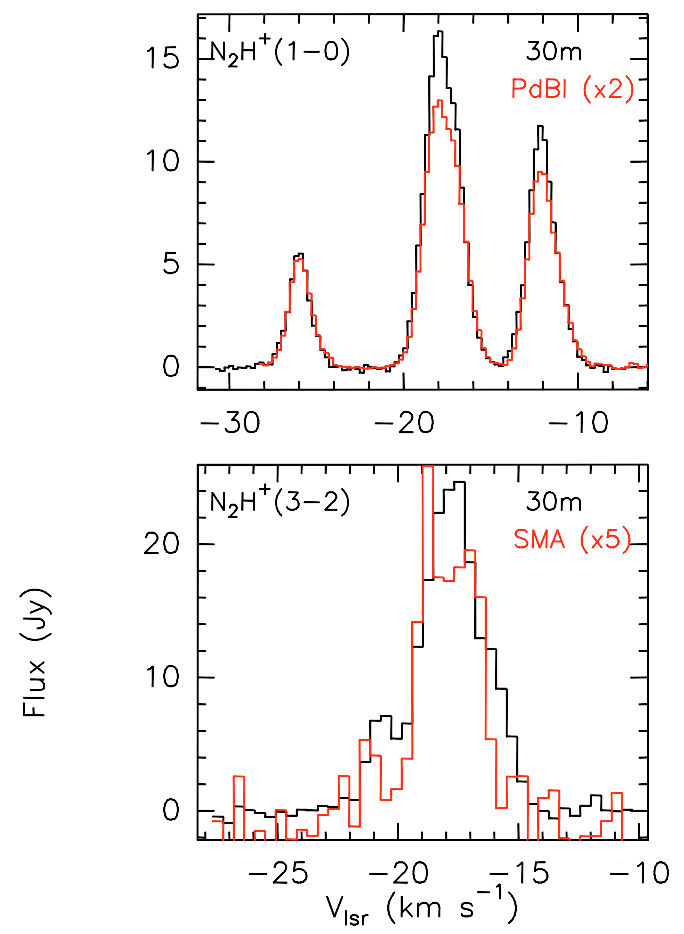

Fig. 10. Flux density comparison between IRAM-30 m spectra and interferometric spectra obtained in the $\mathrm{N}_{2} \mathrm{H}^{+}(1-0)$ (top panel) and $\mathrm{N}_{2} \mathrm{H}^{+}$(3-2) (bottom panel) lines. The interferometric flux density of the $\mathrm{N}_{2} \mathrm{H}^{+}(1-0)$ line, measured with the PdBI, has been multiplied by a factor of 2, while that of the $\mathrm{N}_{2} \mathrm{H}^{+}$(3-2) line, measured with the SMA, by a factor of 5. Both PdBI and SMA spectra have been obtained integrating the maps in Figs. 1 and 2 of Paper I over the $3 \sigma$ level.

with the IRAM-30 m telescope (see Fontani et al. 2006). We have resampled the single-dish spectra of $\mathrm{N}_{2} \mathrm{H}^{+}(1-0)$ and (3-2) to the same resolution in velocity as the interferometric spectra. The superposition of the spectra is shown in Fig. 10: in the $\mathrm{N}_{2} \mathrm{H}^{+}(1-0)$ line, the flux measured by the PdBI is $\sim 2$ times less than that measured with the IRAM-30 m telescope, while in the $\mathrm{N}_{2} \mathrm{H}^{+}$(3-2) line the flux measured with the SMA is only one fifth of that measured with the $30 \mathrm{~m}$ antenna. This indicates that the extended emission is much more resolved in the SMA map of the (3-2) line than in the PdBI map of the (1-0) line.

\subsubsection{Velocity field}

From the molecular lines observed one can derive information about the velocity field in the source. In particular, the peak velocity, $V_{\mathrm{LSR}}$, of the lines gives information on ordered motion of the gas (rotation, inward or outward motion), while the line widths, $\Delta V$ provide information on the gas turbulent motion. In this section, we show the results obtained from the $\mathrm{N}_{2} \mathrm{H}^{+}(1-0)$ line, for which the spectra are not affected by central dips as are those of ${ }^{12} \mathrm{CO}$ and its isotopologues. Also, this tracer is fairly well detected towards both the continuum cores and the deuterated ones, so that it allows us to investigate both ordered and turbulent motion across the whole source.

To derive $V_{\mathrm{LSR}}$ and $\Delta V$, we adopted the following procedure: from the channel maps of the $\mathrm{N}_{2} \mathrm{H}^{+}(1-0)$ line, we extracted a spectrum from each pixel inside the $3 \sigma$ level of the integrated emission (pixel size $\sim 1^{\prime \prime} \times 1^{\prime \prime}$ ), and then fitted these spectra as described in Sect. 2.4.

In Fig. 11 we show the maps of $\Delta V$ (left panel), and $V_{\mathrm{LSR}}$ (right panel) of the $\mathrm{N}_{2} \mathrm{H}^{+}(1-0)$ line. From the left panel, one 

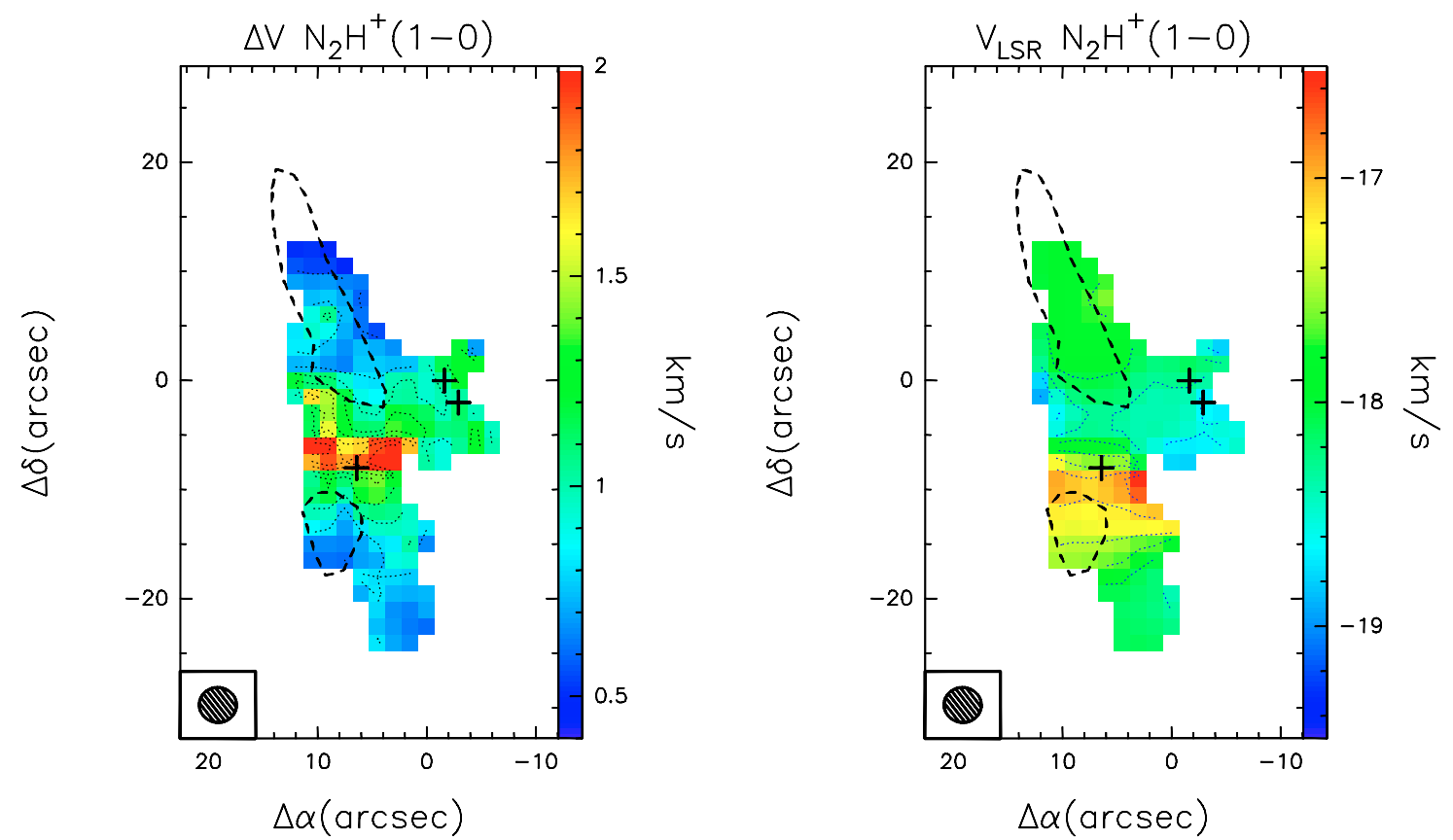

Fig. 11. Left panel: map of the $\mathrm{N}_{2} \mathrm{H}^{+}(1-0)$ line width, $\Delta V$ (coloured-scale). The dotted contours range from 0.4 to $2.0 \mathrm{~km} \mathrm{~s}{ }^{-1}$, in steps of $0.2 \mathrm{~km} \mathrm{~s}^{-1}$. The crosses indicate the position of the continuum sources. The dashed lines represent the $3 \sigma$ level of N and S. The PdBI synthesised beam at the frequency of the $\mathrm{N}_{2} \mathrm{H}^{+}(1-0)$ line is shown in the bottom left corner. Right panel: same as top panel for the peak velocity, $V_{\mathrm{LSR}}$, of the $\mathrm{N}_{2} \mathrm{H}^{+}(1-0)$ line. The dotted contours range from -16.8 to $-19.2 \mathrm{~km} \mathrm{~s}^{-1}$, in steps of $0.3 \mathrm{~km} \mathrm{~s}^{-1}$.

can see that the line widths are clearly broader than $\sim 1 \mathrm{~km} \mathrm{~s}^{-1}$ in the region where the continuum cores $\mathrm{C} 1$ and $\mathrm{C} 2$ and the $\mathrm{N}_{2} \mathrm{H}^{+}(1-0)$ main peaks are located. On the other hand, the gas is more quiescent around this central region, and towards the position of $\mathrm{N}$ and $\mathrm{S}\left(\Delta V\right.$ between $\sim 0.4$ and $1 \mathrm{~km} \mathrm{~s}^{-1}$ ). These results indicate that in the continuum cores, as well as in the $\mathrm{N}_{2} \mathrm{H}^{+}$main peaks, the star formation process is actively taking place, while in the deuterated cores the gas is more quiescent because they are still in the pre-stellar phase. Interestingly, the plot in the right panel of Fig. 11 indicates that in the region where the turbulence is higher the gas is also red-shifted with respect to the systemic velocity, while no clear trend is seen in the other regions of the source, in which the gas velocity is quite close to the systemic one. This is probably caused by the interaction with the red lobe of the ${ }^{12} \mathrm{CO}$ outflow, which is expanding in that portion of the cluster.

In Sect. 5.2, we will discuss in more detail this interaction, and how $\Delta V$ and $V_{\text {LSR }}$ vary in the deuterated cores $\mathrm{N}$ and $\mathrm{S}$, by also using the $\mathrm{N}_{2} \mathrm{D}^{+}(3-2)$ line.

\subsection{Outflow parameters from ${ }^{12} \mathrm{CO}$ line wings}

In Table 5, the characteristics of the flow are given. Column 1 lists the computed parameters which are: the $\mathrm{H}_{2}$ column density of the blue- and red-lobe, $N_{\mathrm{b}}$ and $N_{\mathrm{r}}$; the total mass, $M_{\text {out }}$; the momentum, $P_{\text {out }}$; the energy, $E$; the dynamical timescale, $t_{\text {dyn }}$; the mass entrainment rate, $\dot{M}_{\text {out }}$; the mechanical force, $\dot{P}_{\text {out }}$; the mechanical luminosity, $L_{\mathrm{m}}$.

The $\mathrm{H}_{2}$ column densities in both the blue and red lobe, $N_{\mathrm{b}}$ and $N_{\mathrm{r}}$, have been derived from the ${ }^{12} \mathrm{CO}(2-1)$ line integrated emission in the wings according to the standard relations (see e.g. Rohlfs \& Wilson 2004):

$N_{\mathrm{b}, \mathrm{r}}=\frac{\mathrm{H}_{2}}{\mathrm{CO}} \frac{N_{J(\mathrm{~b}, \mathrm{r})}}{g_{J}} Q \exp \left(E_{J} / T_{\mathrm{ex}}\right)$,
Table 5. Physical parameters of the ${ }^{12} \mathrm{CO}$ outflow.

\begin{tabular}{cccc}
\hline \hline & ${ }^{12} \mathrm{CO}(2-1)$ & & \\
parameter & no corr. & $\theta=57^{\circ}$ & $\theta=80^{\circ}$ \\
\hline$N_{\mathrm{b}}\left(\times 10^{21} \mathrm{~cm}^{-2}\right)$ & 7.7 & & \\
$N_{\mathrm{r}}\left(\times 10^{21} \mathrm{~cm}^{-2}\right)$ & 9.7 & & \\
$M_{\text {out }}\left(M_{\odot}\right)$ & 1.9 & & \\
$P_{\text {out }}\left(M_{\odot} \mathrm{km} \mathrm{s}^{-1}\right)$ & 12 & 22 & 70 \\
$E\left(\times 10^{45} \mathrm{erg}\right)$ & 1.1 & 3.6 & 36 \\
$t_{\text {dyn }}\left(\times 10^{3} \mathrm{yr}\right)$ & 11.8 & 7.65 & 2.08 \\
$\dot{M}_{\text {out }}\left(\times 10^{-4} M_{\odot} \mathrm{yr}^{-1}\right)$ & 1.6 & 2.5 & 9.1 \\
$\dot{P}_{\text {out }}\left(\times 10^{-3} M_{\odot} \mathrm{km} \mathrm{s}^{-1} \mathrm{yr}^{-1}\right)$ & 1.0 & 2.9 & 34 \\
$L_{\mathrm{m}}\left(L_{\odot}\right)$ & 0.755 & 3.9 & 140 \\
\hline
\end{tabular}

$N_{\mathrm{J}(\mathrm{b}, \mathrm{r})}=\frac{3 k}{8 \pi v \mu^{2}} \frac{2 J+1}{J} \frac{\tau}{1-\exp (-\tau)} \int_{\mathrm{b}, \mathrm{r}} T_{\mathrm{MB}} \mathrm{d} V$.

In Eq. (2), $\frac{\mathrm{H}_{2}}{\mathrm{CO}}$ is the inverse relative abundance of $\mathrm{CO}$ (assumed to be $10^{4}$, Frerking et al. 1982), $N_{J}, g_{J}$ and $E_{J}$ are the column density, the statistical weight and the energy (in K) of the upper level (i.e. $J=2$ ), respectively, $Q$ is the partition function and $T_{\text {ex }}$ the excitation temperature. For this latter, we have assumed the dust temperature, i.e. $30 \mathrm{~K}$, assuming coupling between gas and dust. In Eq. (3), $k$ is the Boltzmann constant, $\mu$ the dipole moment (0.11 Debye for CO), $v$ the line rest frequency, $\tau$ the optical depth and $\int T_{\mathrm{MB}} \mathrm{d} V$ is the integrated intensity in the line wings. The values listed in Table 5 have been derived assuming optically thin emission, i.e. $\frac{\tau}{1-\exp (-\tau)} \sim 1$. By comparing the ${ }^{12} \mathrm{CO}$ and ${ }^{13} \mathrm{CO}$ emission in the wings, we have found that this assumption is correct for our data.

All the other parameters given in Table 5 have been derived according to Zhang et al. (2005), taking into account correction for the flow inclination angle, $\theta$, with respect to the line-of-sight. The parameters are given for no correction for the inclination angle (Col. 2), for $\theta=57^{\circ}$ (the mean inclination angle given by 
Cabrit \& Bertout 1986, Col. 3), and for an extreme value of $\theta=$ $80^{\circ}$ (Col. 4). Since the outflow orientation appears to be close to the plane of the sky, we will consider the values computed for $\theta=57^{\circ}$ and $\theta=80^{\circ}$ as lower and upper limits. In this range, the flow parameters (outflow mass, momentum, energy, mechanical force and mechanical luminosity) are consistent with the results obtained for outflows associated with intermediate-/high-mass YSOs observed at high angular resolution (e.g. Molinari et al. 2002; Beuther et al. 2004, 2006).

We will further discuss the nature of the exciting source of the CO outflow in Sect. 5.1.

\section{Discussion}

\subsection{Nature of the continuum sources}

The maps shown in Sect. 3.1 and the parameters derived in Sect. 4 allow us to discuss the nature of the millimeter continuum cores $\mathrm{C} 1-\mathrm{b}, \mathrm{C} 1-\mathrm{a}$ and $\mathrm{C} 2$.

C1-b probably hosts a very young early-B ZAMS star. This is indicated by several pieces of evidence: first, Molinari et al. (2002) have detected towards I05345 a cm-continuum source whose position roughly corresponds to that of $\mathrm{C} 1-\mathrm{b}$, and the Lyman continuum derived from their observations suggests that the embedded object is a newly formed B2-B3 ZAMS star. Second, the source is embedded in a hot core, as demonstrated by the kinetic temperature derived from $\mathrm{CH}_{3} \mathrm{CN}$ (12-11) in Sect. 4.1, and by the presence of other high-excitation lines (see Fig. 6), and it lies where the main peak of the mid-infrared $24 \mu \mathrm{m}$ continuum emission is detected (see Fig. 3). Third, it is close to the center of the outflow lobes detected in ${ }^{12} \mathrm{CO}$ (2-1) (Fig. 5), even though a significant contribution to the observed blue- and red-shifted emission can arise from $\mathrm{C} 1$-a and, less likely, from another source not seen in the continuum image, located at the position of the western emission peak of $\mathrm{N}_{2} \mathrm{H}^{+}$(see Fig. 5). As discussed in Sect. 4.2, even though the masses derived form the millimeter continuum are 5.2 and $0.6 M_{\odot}$, these measurements represent the mass of the circumstellar gas only, and thus they do not contrast with the previous findings.

The source embedded in C1-a is probably a very young intermediate-mass protostar. This is indicated by: the core location at the centre of the ${ }^{12} \mathrm{CO}$ outflow lobes, which suggests that the object embedded in the core is one of the main sources driving the powerful outflow; the core gas mass listed in Table 3; the non-detection of the core in the centimeter continuum (Molinari et al. 2002), indicating an evolutionary stage prior to the formation of an HII region. Based on these findings, we believe that the source embedded in $\mathrm{C} 1-\mathrm{a}$ is an intermediate-mass protostar still in the main accretion phase (i.e. a Class 0 object).

The nature of the source embedded in $\mathrm{C} 2$ is unclear. The core mass (see Table 3) and the non-detection in the $24 \mu \mathrm{m}$ image suggest that it can be an intermediate-mass embedded protostar. The source is not associated with a detectable outflow, which however could be an indication that the embedded source is extremely young. Interestingly, one can notice from Fig. 4 that $\mathrm{C} 2$ is in the middle of a filamentary structure extended in the N-S direction which also contains the deuterated cores $\mathrm{N}$ and $\mathrm{S}$, and four of the five peaks of the $\mathrm{N}_{2} \mathrm{H}^{+}(1-0)$ integrated emission. This may indicate that this portion of the source hosts the youngest low- and intermediate-mass objects of the protocluster, and it is consistent with theoretical models that predict that filamentary structures are common morphologies of molecular clouds in several classes of dynamical formation models (e.g. van Loo 2007).

\subsubsection{A proto-Trapezium system?}

From the positions given in Table 3, we derive that the projected linear separation among the three continuum sources goes from $\sim 0.014 \mathrm{pc}$ between $\mathrm{C} 1-\mathrm{a}$ and $\mathrm{C} 1-\mathrm{b}$, to $\sim 0.07 \mathrm{pc}$ between $\mathrm{C} 1-\mathrm{a}$ and $\mathrm{C} 2$, i.e. from $\sim 2800$ to $14000 \mathrm{AU}$, at the given distance. Looking at the 284 and $225 \mathrm{GHz}$ images in Fig. 2, one could also suggest the possible presence of a fourth continuum unresolved source to the NW of $\mathrm{C} 1-\mathrm{a}$. This is suggested by the elongated shape of $\mathrm{C} 1-\mathrm{a}$ in this direction, while the synthesised beam of the SMA at $284 \mathrm{GHz}$ is elongated in the NE-SW direction. Assuming spherical symmetry, the stellar density in this region is thus $\sim 2.3 \times 10^{4}$ proto-stars $\mathrm{pc}^{-3}$, while it is $\sim 1.7 . \times 10^{4}$ proto-stars $\mathrm{pc}^{-3}$ assuming the three resolved sources only. These values are consistent with the stellar density measured in the Trapezium cluster in Orion, estimated to be of

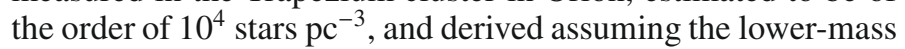
stars in the cluster as well. This suggests that the stellar density of $\sim 1.7 \times 10^{4}$ proto-stars $\mathrm{pc}^{-3}$ is a lower limit, and that perhaps the three young intermediate-/high-mass (proto-)stars embedded in $\mathrm{C} 1-\mathrm{a}, \mathrm{C} 1-\mathrm{b}$ and $\mathrm{C} 2$ (plus possibly the unresolved one to the NW of C1-a) represents a Trapezium-like system in the making.

Similar multiple systems of forming intermediate-/highmass stars have been discovered recently through high-angular resolution observations (see e.g. Beuther et al. 2007b; Rodon et al. 2008), although these systems are denser than the one in

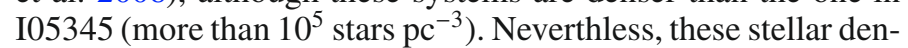

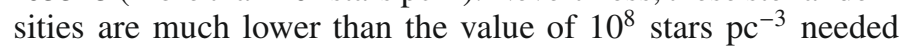
for the formation of high-mass stars through merging of several lower-mass stars at the center of rich clusters (see e.g. Bonnell et al. 1998), but comparable to the value sufficient to produce binary induced mergers (Bonnell \& Bate 2005).

\subsection{Nature of the deuterated cores}

In Paper I, we discussed the nature of the deuterated cores based on the dust continuum emission and the deuterium fractionation derived from the column density ratio $N\left(\mathrm{~N}_{2} \mathrm{D}^{+}\right) / N\left(\mathrm{~N}_{2} \mathrm{H}^{+}\right)$. We derived the mass of the cores following two approaches: one from the virial theorem, and the other assuming an average abundance of $\mathrm{N}_{2} \mathrm{H}^{+}$. For completeness, in Col. 6 of Table 4 we list the main results obtained from the second approach in Paper I. These values, together with a $D_{\text {frac }} \simeq 0.1$ (Col. 4 of Table 4 ), suggested that both cores are in the pre-stellar phase but at present they are not massive. On the other hand, as discussed in Sect. 3.3 of Paper I, the average spectra of the $\mathrm{N}_{2} \mathrm{H}^{+}(1-0)$ and $\mathrm{N}_{2} \mathrm{D}^{+}$(3-2) lines in both $\mathrm{N}$ and $\mathrm{S}$ show lines broader than those typically observed in low-mass pre-stellar cores.

We proposed two possible scenarios for the nature of the two $\mathrm{N}_{2} \mathrm{D}^{+}$condensations: (i) they are quiescent low-mass starless cores, and therefore they are going to form low-mass stars (in particular, $\mathrm{N}$ is likely to harbor several unresolved cores); (ii) they are condensations still in dynamic evolution and may be changing their mass and shape, undergoing either fragmentation or accretion from the parental cloud. In particular, the second scenario is suggested by the predictions of the "competitive accretion models", which predict formation of massive stars in a clustered environment through dynamical interaction among several "seeds" of forming (proto-)stars (see e.g. Zinnecker \& Yorke 2007, for a review).

To better understand the nature of the two condensations, a detailed analysis of the gas kinematics is certainly very helpful. For this reason, here we investigate in more detail the gas kinematics in $\mathrm{N}$ and $\mathrm{S}$ by using as diagnostics the peak velocity and 

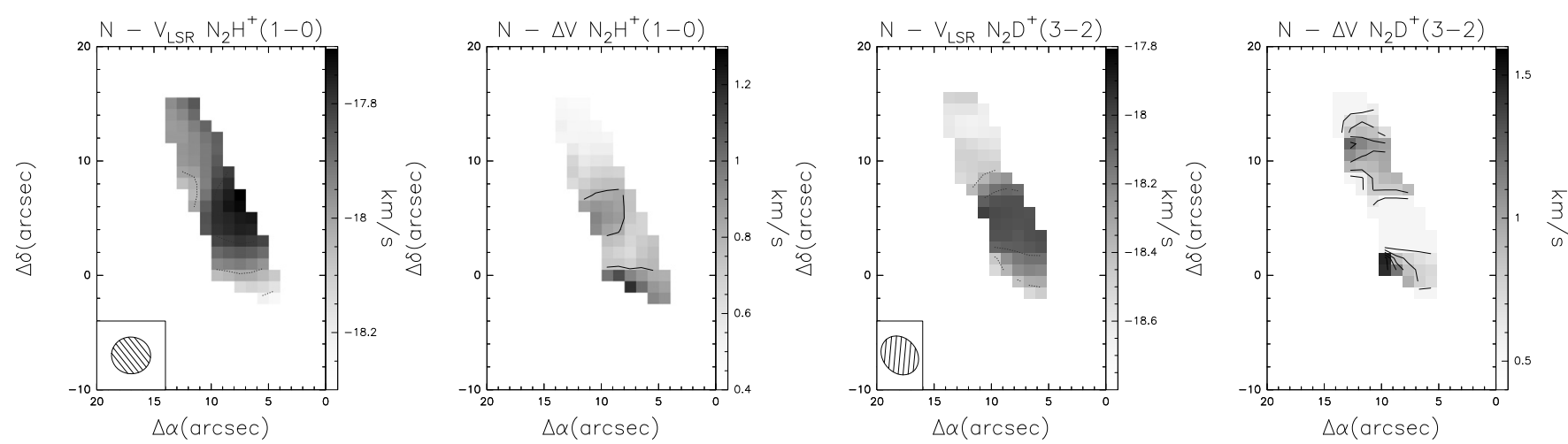

Fig. 12. Left panels: map of the peak velocity $\left(V_{\mathrm{LSR}}\right)$ and line width $(\Delta V)$ derived from the $\mathrm{N}_{2} \mathrm{H}^{+}(1-0)$ line inside the $3 \sigma$ level of the $\mathrm{N}_{2} \mathrm{D}^{+}(3-2)$ emission of core $\mathrm{N}$. The vertical grey-scale on the right side of the two plots indicates the intensity, in $\mathrm{km} \mathrm{s}^{-1}$, of $V_{\mathrm{LSR}}$ and $\Delta V$, respectively. In the bottom left corner, the synthesised beam of the channel maps are shown. Right panels: same as left panel for the $\mathrm{N}_{2} \mathrm{D}^{+}(3-2)$ line.
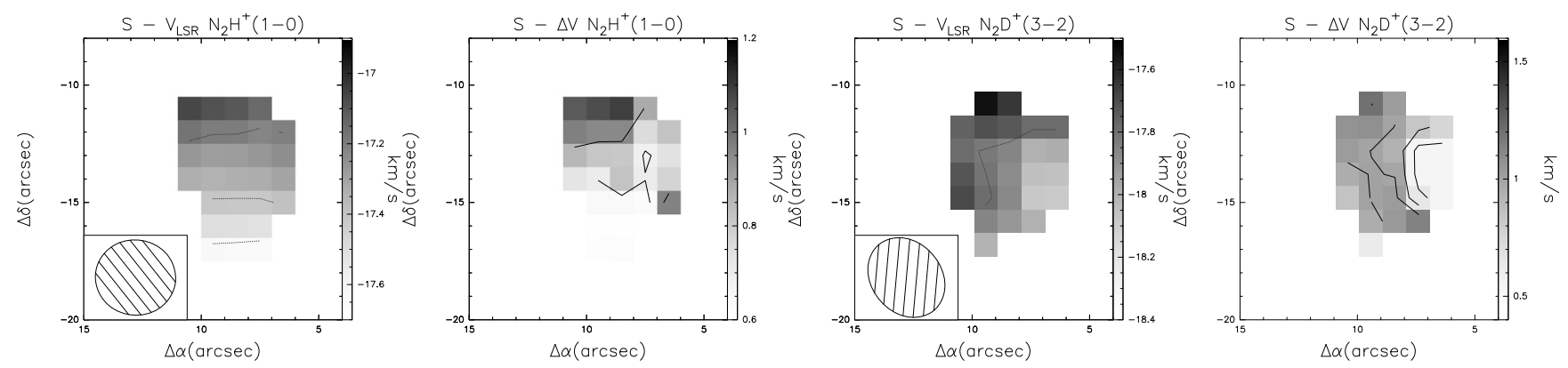

Fig. 13. Same as Fig. 12 for core S.

widths of the $\mathrm{N}_{2} \mathrm{H}^{+}(1-0)$ and $\mathrm{N}_{2} \mathrm{D}^{+}$(3-2) lines, following the same approach described in Sect. 4.3.2.

\subsubsection{Condensation $\mathrm{N}$}

In the two left panels of Fig. 12 we show the maps of $V_{\text {LSR }}$ and $\Delta V$, respectively, obtained for $\mathrm{N}$ from the $\mathrm{N}_{2} \mathrm{H}^{+}(1-0)$ line. The same plots derived from $\mathrm{N}_{2} \mathrm{D}^{+}(3-2)$ are shown in the two right panels. One can see in the first place that the velocity field in this core is quite complex. The line width of the $\mathrm{N}_{2} \mathrm{H}^{+}(1-0)$ line are slighly larger in the southern region of the core, while that of the $\mathrm{N}_{2} \mathrm{D}^{+}(3-2)$ looks larger in the northern and, less prominently, in the south-eastern portion of the core. For both species, the values range from $\sim 0.6$ to $\sim 1.4 \mathrm{~km} \mathrm{~s}^{-1}$, and are on average 3-4 times larger than those found in low-mass pre-stellar cores (see e.g. Caselli et al. 2002a; for the case of L1544, Crapsi et al. 2005). However, for the $\mathrm{N}_{2} \mathrm{D}^{+}$(3-2) line, in the positions where $\Delta V \sim 0.5 \mathrm{~km} \mathrm{~s}^{-1}$, these values can be influenced by the poor spectral resolution of $\sim 0.5 \mathrm{~km} \mathrm{~s}^{-1}$ (see Table 1). Therefore, it is possible that linewidths smaller than $0.5 \mathrm{~km} \mathrm{~s}^{-1}$ are present but not observable with the adopted spectral resolution. The peak velocity of both $\mathrm{N}_{2} \mathrm{H}^{+}$and $\mathrm{N}_{2} \mathrm{D}^{+}$lines is between -17.7 and $\sim-18.4 \mathrm{~km} \mathrm{~s}^{-1}$, i.e. close to the systemic velocity, but the gas in the southern region of the core looks slightly red-shifted with respect to the northern one. Also, from Fig. 5 one can notice that the red lobe of the ${ }^{12} \mathrm{CO}$ outflow, likely driven by $\mathrm{C} 1-\mathrm{b}$, arises from a region spatially coincident with the southern portion of core $\mathrm{N}$, and possibly interacts with it: the gas in the red-shifted lobe of the outflow probably flows behind the core, undergoing drag on part of the southern gas of $\mathrm{N}$. In this way, one can explain the observed red-shift of the $\mathrm{N}_{2} \mathrm{H}^{+}$and $\mathrm{N}_{2} \mathrm{D}^{+}$lines in the southern portion of $\mathrm{N}$. This could also be interpreted as being due to the presence of unresolved low-mass cores at a different velocity, and the $\mathrm{N}_{2} \mathrm{H}^{+}$emission peaks shown in Fig. 4 are consistent with this interpretation. In this case, the observed broad lines are just due to the superposition of the different cores. However, this seems very unlikely since the red-shifted gas in the southern portion of the core is spatially coincident with the bulk of the red lobe of the ${ }^{12} \mathrm{CO}$ outflow. We suggest that the observed redshifted emission and broad lines in the southern portion of $\mathrm{N}$ are both due to the interaction with the ouflow.

\subsubsection{Condensation $S$}

In Fig. 13 we show the same maps as in Fig. 12 for core S. The peak velocity of both the $\mathrm{N}_{2} \mathrm{H}^{+}(1-0)$ and $\mathrm{N}_{2} \mathrm{D}^{+}(3-2)$ lines look red-shifted towards the north of the core (which appears to be more shifted to the north-east in $\mathrm{N}_{2} \mathrm{D}^{+}$), suggestive of a rotation motion. A disk-like structure has been observed in the low-mass pre-stellar core L1544, and it is expected to be caused by the ambipolar diffusion mechanism (Ciolek \& Basu 2000; Caselli et al. 2002a). However, L1544 also looks flatter than what is seen in core $\mathrm{S}$, which shows a spherical shape. This can be due to a different orientation with respect to the line of sight and to the worse angular resolution of our data, since Caselli et al. (2002a) obtained observations with linear resolutions a factor of $\sim 3$ better than ours. Interestingly, $\Delta V$ of both $\mathrm{N}_{2} \mathrm{H}^{+}$and $\mathrm{N}_{2} \mathrm{D}^{+}$ are larger towards the north-eastern part of the core, so that the red-shifted gas also appears to be more turbulent. As for N, this is suggestive of an interaction of the core with the red lobe of the ${ }^{12} \mathrm{CO}$ outflow, the southern edge of which touches the upper side of core $S$ (see the upper panel of Fig. 5).

In summary, the shape and the mass of this core indicate that it is a low-mass pre-stellar core, but the high turbulence and the 
red-shifted gas revealed in the northern part of the core (probably triggered by the neighbouring ${ }^{12} \mathrm{CO}$ outflow) can influence the evolution of the core itself.

\subsubsection{General conclusion on the nature of $\mathrm{N}$ and $\mathrm{S}$}

The main finding of the analysis performed above is that both cores are characterised by a probable interaction with the red lobe of the ${ }^{12} \mathrm{CO}$ outflow. This can trigger turbulence in the cores themselves and can influence their evolution. In theoretical models of clustered star formation, turbulence (which in this case is generated by already formed protostars) can create density modifications across the cloud, generating several dense and cold "seeds", which subsequently can accrete backgound gas that was initially not associated with the "accretion domain" of the seed itself (see e.g. Bonnell et al. 2004; and McKee \& Tan 2003). In this scenario, the two condensations can become more massive and form pre-stellar cores of higher mass. Alternatively, the interaction with the other cluster members, in particular with the powerful ${ }^{12} \mathrm{CO}$ outflow, could also cause the fragmentation of the condensations.

\subsection{Comparison with other star forming regions}

\subsubsection{Intermediate-/high-mass}

The analysis made in Sects. 5.2 and 5.1 indicates that in I05345 there are objects with masses between $\sim 2$ and $\sim 20 M_{\odot}$, and in different evolutionary stages: pre-stellar core candidates, intermediate-mass class 0 protostars, and a newly formed early$\mathrm{B}$ ZAMS star. In recent years, many star forming regions harboring intermediate- and high-mass young stellar objecs have been investigated at high-angular resolution, and several of these regions show a complex structure as seen in I05345. However, only in very few of them has evidence of the presence of prestellar core candidates been found.

Among the studies performed so far, I05345 appears to have the strongest similarities with the high-mass protocluster associated with IRAS 20343+4129. Palau et al. (2007) have observed this cluster with the SMA in the sub-mm continuum and in ${ }^{12} \mathrm{CO}(2-1)$. Since the synthesised beam and the source distance are $\sim 3^{\prime \prime}$ and $1.4 \mathrm{kpc}$, respectively, their observations have a linear resolution comparable to our ones. They found two intermediate- to high-mass young stellar objects, one of which is driving a molecular outflow, and the other one is driving an expanding cavity. Interestingly, the gas at the edge of the cavity seems to be compressed into several dense cores, detected in the millimeter continuum only. Palau et al. (2007) propose that these are potential starless cores representing a future generation of stars. In this scenario, the formation of new stars in the cluster is caused by the interaction between the highest mass objects (which are expected to form first at cluster center), and the residual circumstellar (circum-cluster) material as in I05345.

Leurini et al. (2007) have investigated at high-angular resolution the high-mass star forming region IRAS $05358+3543$, and found 4 massive millimeter cores. One of these seems to be in the pre-stellar phase, being very cold $(T \leq 20 \mathrm{~K})$ and with a large reservoir of material $\left(M \sim 19 M_{\odot}\right)$. However, the core does not show any interaction with the other cluster members, and the authors suggest that it could be also a very embedded massive protostar.

\subsubsection{Low-mass}

Several well-studied low-mass star forming regions show filamentary structures similar to that seen in I05345, and clustered star formation.

In the $\rho$-Ophiuchi molecular cloud complex, the large majority of low-mass millimeter cores associated with both pre- and proto-stellar objects are found to be distributed in filaments (see e.g. Nutter et al. 2006; André et al. 2007). In particular, the structure of one of these filaments, Oph A, closely resembles that of core N. André et al. (2007) have used $\mathrm{N}_{2} \mathrm{H}^{+}$(1-0) to perform a detailed study of the kinematics in Oph $\mathrm{A}$, and their results are different from ours: first, they derive an average line width (thermal + non-thermal component) of $\sim 0.4 \mathrm{~km} \mathrm{~s}^{-1}$, which is yet more than 2 times smaller than that measured in our deuterated cores, even though in the $\mathrm{N}_{2} \mathrm{D}^{+}$lines we are limited by a spectral resolution of $0.5 \mathrm{~km} \mathrm{~s}^{-1}$, and we are at the limit of our resolution towards some parts of the deuterated cores. Second, they do not find any evidence of interactions between the different cores, so that there is not feedback from protostellar activity. Similar morphologies have been obtained observing $\mathrm{N}_{2} \mathrm{H}^{+}(1-0)$ towards the Perseus molecular cloud by Kirk et al. (2007), in which there seem to be a minimal contribution of turbulence in the observed line widths.

These results clearly indicate that pre-stellar cores in lowmass star forming regions are commonly found in filaments similarly to what we have found in I05345, but they are much less turbulent and interact less with the other cluster members than those studied in this work. Therefore, as suggested in Sect. 5.2, in the condensations associated with I05345 the nearby intermediate- and high-mass young objects likely play an important role in triggering turbulence and, eventually, in causing either the growth or the dispersion of the gas in these cores.

\section{Summary and conclusions}

We have presented a full report of the observations partially analysed by Fontani et al. (2008, Paper I) towards the intermediate/high-mass star forming region IRAS $05345+3157$. The observations were performed with the PdBI and SMA in the following molecular transitions: $\mathrm{N}_{2} \mathrm{H}^{+}(1-0)$ with $\mathrm{PdBI}, \mathrm{N}_{2} \mathrm{H}^{+}$(3-2) and $\mathrm{N}_{2} \mathrm{D}^{+}$(3-2), both with the SMA. At the frequencies of these lines, we have simultaneously observed the continuum emission, as well as other rotational transitions such as ${ }^{12} \mathrm{CO}(2-1)$, ${ }^{13} \mathrm{CO}(2-1), \mathrm{C}^{18} \mathrm{O}(2-1)$ and a few other high excitation lines of less abundant species. In Paper I, the main finding presented was the detection of two molecular condensations (called $\mathrm{N}$ and $\mathrm{S}$ ) showing high values of deuterium fractionation $(\sim 0.1)$, derived from the column density ratio $N\left(\mathrm{~N}_{2} \mathrm{D}^{+}\right) / N\left(\mathrm{~N}_{2} \mathrm{H}^{+}\right)$.

In this work, the following main results have been obtained:

- The continuum emission at 96 and $225 \mathrm{GHz}$ reveals two main cores, $\mathrm{C} 1$ and $\mathrm{C} 2$, and $\mathrm{C} 1$ is resolved into two components in the image at $284 \mathrm{GHz}$, called C1-a and C1-b. C1 is coincident with a prominent mid-infrared emission detetcted in the Spitzer MIPS images at 24 and $70 \mu \mathrm{m}$, which is only barely detected towards $\mathrm{C} 2$.

- The integrated intensity of the optically thin component of the $\mathrm{N}_{2} \mathrm{H}^{+}$(1-0) line shows an extended distribution with 5 emission peaks. Two of them fall inside condensation $\mathrm{N}$, another one at the edge of condensation $\mathrm{S}$, while none of them overlaps with the continuum sources.

- The integrated emission in the ${ }^{12} \mathrm{CO}(2-1)$ line wings reveals the presence of a powerful bipolar outflow oriented roughly 
in the WE direction. From the outflow geometry, the sources $\mathrm{C} 1-\mathrm{a}$ and $\mathrm{C} 1-\mathrm{b}$ are the best candidates for powering the outflow. Assuming a unique source driving the outflow, its parameters are consistent with an engine which is a high-mass young stellar object.

- The $\mathrm{N}_{2} \mathrm{H}^{+}(1-0)$ line widths are between 1 and $2 \mathrm{~km} \mathrm{~s}^{-1}$ in a region that spatially corresponds to that where the continuum cores are located, while they are significantly smaller (between 0.5 and $1.5 \mathrm{~km} \mathrm{~s}^{-1}$ ) in the deuterated cores.

- Based on previous observations and on the results presented in this work, we can conclude that $\mathrm{C} 1$-b very likely harbors a newly formed early-B star embedded inside a hot-core, and $\mathrm{C} 1$-a harbors an intermediate-mass class 0 protostar. The nature of $\mathrm{C} 2$ is unclear but it could be a very embedded intermediate-mass protostar. If we consider the system of these three continuum sources and roughly assume a spherical symmetry, we deduce a star density of $\sim 10^{4}$ stars pc $^{-3}$, consistent with the stellar density of the Trapezium cluster and much lower than the value required to form massive stars through collisions of low-mass ones.

- The nature of the deuterated cores has been discussed mainly using the information on the gas kinematics that one can derive from the lines of $\mathrm{N}_{2} \mathrm{H}^{+}$and $\mathrm{N}_{2} \mathrm{D}^{+}$. Core $\mathrm{S}$ is likely a single low-mass pre-stellar core, and the velocity of both the $\mathrm{N}_{2} \mathrm{H}^{+}$and $\mathrm{N}_{2} \mathrm{D}^{+}$lines indicates a rotation motion roughly in the N-S direction. This motion and the high turbulence measured at the northern edge of the core is likely due to dynamical interaction with the red lobe of the ${ }^{12} \mathrm{CO}$ powerful outflow. The nature of core $\mathrm{N}$ is less clear. Two scenarios are possible: it can be a filament of unresolved low-mass prestellar cores, similarly to Oph A, and in this case the high values of the $\mathrm{N}_{2} \mathrm{H}^{+}$and $\mathrm{N}_{2} \mathrm{D}^{+}$line widths are due to the superposition of the several unresolved cores. Alternatively, it can be a single object still in dynamic evolution, which actively interacts with the red lobe of the ${ }^{12} \mathrm{CO}$ outflow. In this case, the triggered trubulence can cause either the growth or the fragmentation of the core itself.

From these observations, it is not at all clear how the interaction with the other cluster members can make the condensations evolve towards the formation of low- or high-mass objects. Only higher resolution kinematic studies of the large scale gas around these condensations, and the comparison of these results with hydrodynamic evolution models, will help to understand the core evolution and provide important constraints on the dynamical evolution of intermediate-/high-mass star forming regions.

Acknowledgements. Many thanks to the anonymous referee for his/her useful comments and suggestions. It is a pleasure to thank the staff of the Smithsonian Astrophysical Observatory for the SMA observations. We also thank the IRAM staff and Riccardo Cesaroni for their help in the calibration of the PdBI data. F.F. is deeply grateful to Marc Audard, Carla Baldovin and Andres Carmona for useful discussion, and to Keping Qiu for fitting the $\mathrm{CH}_{3} \mathrm{CN}$ spectrum. P.C. thanks Jonathan Tan for useful discussions on protocluster dynamics. F.F. acknowledges support by Swiss National Science Foundation grant (PP002 - 110504). T.B. acknowledges support from US National Science Foundation grant (0708158).

\section{References}

André, Ph., Belloche, A., Motte, F., \& Peretto, N. 2007, A\&A, 472, 519

Bachiller, R., Perez Gutierrez, M., Kumar, M. S. N., \& Tafalla, M. 2001, A\&A, 372,899

Beltràn, M. T., Estalella, R., Girart, J. M., Ho, P. T. P., \& Anglada, G. 2008, A\&A, 481, 93

Bergin, E. A., Goldsmith, P. F., Snell, R. L., \& Ungerechts, H. 1994, ApJ, 431, 674
Beuther, H., \& Sridharan, T. K. 2007, ApJ, 668, 348

Beuther, H., Schilke, P., Sridharan, T. K., et al. 2002, A\&A, 383, 892

Beuther, H., Schilke, P., \& Gueth, F. 2004, ApJ, 608, 330

Beuther, H., Zhang, Q., Sridharan, T. K., Lee, C.-F., \& Zapata, L. A. 2006, A\&A, 454, 221

Beuther, H., Churchwell, E. B., McKee, C. F., \& Tan, J. C. 2007a, Protostars and Planets V, 165

Beuther, H., Zhang, Q., Bergin, E. A., et al. 2007b, A\&A, 468, 1045

Bonnell, I. A., \& Bate, M. R. 2005, MNRAS, 362, 915

Bonnell, I. A., Bate, M. R., \& Zinnecker, H. 1998, MNRAS, 298, 93

Bonnell, I. A., Vine, S. G., \& Bate, M. R. 2004, MNRAS, 349, 735

Cabrit, S., \& Bertout, C. 1986, ApJ, 307, 313

Caselli, P., Myers, P. C., \& Thaddeus, P. 1995, ApJ, 455, L77

Caselli, P., Walmsley, C. M., Zucconi, A., et al. 2002a, ApJ, 565, 331

Caselli, P., Walmsley, C. M., Zucconi, A., et al. 2002b, ApJ, 565, 344

Chakrabarti, S., \& McKee, C. F. 2005, 631, 792

Ciolek, G. E., \& Basu, S. 2000, ApJ, 529, 925

Crapsi, A., Caselli, P., Walmsley, C. M., et al. 2005, ApJ, 619, 379

Fontani, F., Beltran, M. T., Brand, J., et al. 2005, A\&A, 432, 921

Fontani, F., Caselli, P., Crapsi, A., et al. 2006, A\&A, 460, 709

Fontani, F., Caselli, P., Bourke, T. L., Cesaroni, R., \& Brand, J. 2008, A\&A, 477, L45

Frerking, M. A., Langer, W. D., \& Wilson, R. W. 1982, ApJ, 262, 590

Hill, T., Thompson, M. A., Burton, M. G., et al. 2006, MNRAS, 368, 1223

Ho, P. T. P., Moran, J. M., \& Lo, K. Y. 2004, ApJ, 616, L1

Kirk, H., Johnstone, D., \& Tafalla, M. 2007, ApJ, 668, 1042

Kramer, C., Alves, J., Lada, C., et al. 1998, A\&A, 329, L33

Krumholz, M. R., \& McKee, C. F. 2008, Nature, 451, 1082

Kuiper, T. B. H., Rodriguez-Kuiper, E. N., \& Dickinson, D. F. 1984, ApJ, 276, 211

Kurtz, S., Cesaroni, R., Churchwell, E., Hofner, P., \& Walmsley, C. M. 2000, Protostars and Planets IV, 299

Kurtz, S., Hofner, P., \& Alvarez, C. V. 2004, ApJS, 155, 149

Jijina, J., Myers, P. C., \& Adams, F. C. 1999, ApJS, 125, 161

Kuiper, T. B. H., Langer, W. D., \& Velusamy, T. 1996, ApJ, 468, 761

Leurini, S., Beuther, H., Schilke, P., et al. 2007, A\&A, 475, 925

Mathis, J. S., \& Whiffen, G. 1989, ApJ, 341, 808

McKee, C. F., \& Tan, J. C. 2003, ApJ, 585, 850

McKee, C. F., \& Tan, J. C. 2002, Nature, 416, 59

Molinari, S., Brand, J., Cesaroni, R., \& Palla, F. 1996, A\&A, 308, 573

Molinari, S., Brand, J., Cesaroni, R., \& Palla, F. 2000, A\&A, 355, 617

Molinari, S., Testi, L. Rodriguez, L. F., \& Zhang, Q. 2002, ApJ, 570, 758

Nutter, D., Ward-Thompson, D., \& André, P. 2006, MNRAS, 368, 1833

Oliveira, C. M., Hébrard, G., Howk, J. C., et al. 2003, ApJ, 587, 235

Pagani, L., Daniel, F., \& Dubernet, M. L. 2009, A\&A, 494, 719

Palau, A., Estalella, R., Ho, P. T. P., Beuther, H., \& Beltrán, M. T. 2007, A\&A, 474,911

Pillai, T., Wyrowski, F., Hatchell, J., Gibb, A. G., \& Thompson, M. A. 2007, A\&A, 467, 207

Qi, C. 2005, The MIR Cookbook,

http: //cfa-www . harvard. edu/ cqi/mircook.html

Roberts, H., \& Millar, T. J. 2007, A\&A, 471, 849

Rodon, J. A., Beuther, H., Megeath, S. T., \& van der Tak, F. F. S. 2008, A\&A, 490, 213

Rohlfs, K., \& Wilson, T. L. 2004, 4th rev. and enl., ed. K. Rohlfs, \& T. L. Wilson (Berlin: Springer)

Sandell, G., Wright, M., \& Forster, J. R. 2003, ApJ, 590, 45

Sault, R. J., Teuben, P. J., \& Wright, M. C. H. 1995, ASPC, 77, 433

Slysh, V. I., Kalenskii, S. V., \& ValTts, I. E. 2002, ARep, 46, 49

Sridharan, T. K., Beuther, H., Schilke, P., Menten, K. M., \& Wyrowski, F. 2002, ApJ, 566, 931

Tafalla, M., Myers, P. C., Caselli, P., Walmsley, C. M., \& Comito, C. 2002, ApJ, 569,815

Tafalla, M., Santiago, J., Myers, P. C., et al. 2006, A\&A, 455, 577

Thompson, M. A., Hatchell, J., Walsh, A. J., MacDonald, G. H., \& Millar, T. J. 2006, A\&A, 453, 1003

Townes, C. H., \& Schawlow, A. L. 1975, Microwave spectroscopy (New York: Dover)

van Loo, S., Falle, S. A. E. G., Hartquist, T. W., \& Moore, T. J. T. 2007, A\&A, 471,213

Viti, S., Girart, J. M., Garrod, R., Williams, D. A., \& Estalella, R. 2003, A\&A, 399, 187

Wang, Y., Zhang, Q., Rathborne, J. M., Jackson, J., \& Wu, Y. 2006, ApJ, 651, L125

Zhang, Q., Hunter, T. R., Brand, J., et al. 2005, ApJ, 625, 864

Zhang, Q., Hunter, T. R., Beuther, H., et al. 2007, ApJ, 658, 1152

Zhang, Q., Wang, Y., Pillai, T., \& Rathborne, J. 2009 [arXiv: 0902 . 0647]

Zinnecker, H., \& Yorke, H. W. 2007, ARA\&A, 45, 481 